\title{
CONVOLUTION SINGULAR INTEGRALS ON LIPSCHITZ SURFACES
}

\author{
CHUN LI, ALAN MCINTOSH, AND STEPHEN SEMMES
}

\section{CONTENTs}

1. Introduction

2. Clifford analysis

3. Convolution singular integrals on Lipschitz surfaces

4. Reverse square function estimates

5. Operator bounds

6. Hardy decomposition of $K\left(S_{\mu}^{o}\right)$

7. Condition $(*)$

\section{INTRODUCTION}

Let us recall some results about convolution singular integrals on curves.

There have been many proofs of the $L_{p}$-boundedness of the singular Cauchy integral operator on a Lipschitz curve $\gamma$ in the complex plane $\mathbb{C}$ since this was first proved by Calderón [2] (for curves with small Lipschitz constants) and by Coifman, McIntosh, and Meyer [3] in the general case. In particular two straightforward proofs have recently been given in the paper [5] by Coifman, Jones, and Semmes. In the first of these it is shown that bounds on the Cauchy operator can be obtained directly from quadratic estimates proved by Kenig [11] for those $L_{2}$ functions on $\gamma$ which are in the Hardy spaces $H_{ \pm}^{2}(\gamma)$ consisting of boundary values of holomorphic functions defined on one side of the curve $\gamma$ or the other. Actually simple proofs are also given for those results of Kenig which are needed.

An alternative presentation of this, more in the spirit of functional calculi of operators, is presented in [15], where the following theorem is proved. In it, $\gamma$ denotes a curve of the form $\gamma=\{x+i g(x) \in \mathbb{C}: x \in \mathbb{R}\}$, where $g$ is a Lipschitz function which satisfies $\left\|g^{\prime}\right\|_{\infty} \leq \tan \omega<\infty$, and $S_{\mu}^{o}$ denotes the

Received by the editors December 20, 1990.

1991 Mathematics Subject Classification. Primary 42B20.

The first and second authors were supported by the Australian Government through the Australian Research Council. The third author was supported by the NSF and Sloan Foundation. 
open double sector which consists of those complex numbers $z$ which satisfy $|\arg ( \pm z)|<\mu$, with $\omega<\mu<\pi / 2$, while $S_{\mu+}^{o}$ denotes the component in which $|\arg z|<\mu$.

Theorem 1.1. Suppose that $\phi$ and $\phi_{1}$ are holomorphic functions on $S_{\mu}^{o}$ and $S_{\mu+}^{o}$, that $z \phi(z)$ and $\phi_{1}(z)$ are bounded in $z$, that $\phi_{1}^{\prime}(z)=\phi(z)+\phi(-z)$ for all $z \in S_{\mu+}^{o}$, and that $1<p<\infty$. Then there is a bounded linear operator $T_{\left(\phi, \phi_{1}\right)}$ on $L_{p}(\gamma)$ defined for all $u \in L_{p}(\gamma)$ and almost all $z \in \gamma$ by

$$
\left(T_{\left(\phi, \phi_{1}\right)} u\right)(z)=\lim _{\varepsilon \rightarrow 0+}\left\{\int_{|z-\zeta| \geq \varepsilon} \phi(z-\zeta) u(\zeta) d \zeta+\phi_{1}(\varepsilon \underline{t}(z)) u(z)\right\}
$$

where $\underline{t}(z)$ is the unit tangent vector to $\gamma$ which is defined at almost all $z \in \gamma$. Moreover

$$
\left\|T_{\left(\phi, \phi_{1}\right)} u\right\|_{p} \leq C_{\omega, \mu, p}\left[\sup \left\{|z \phi(z)|: z \in S_{\mu}^{o}\right\}+\sup \left\{\left|\phi_{1}(z)\right|: z \in S_{\mu+}^{o}\right\}\right]\|u\|_{p}
$$

for some constants $C_{\omega, \mu, p}$ which depend only on $\omega, \mu$ and $p$.

Of course the singular Cauchy integral operator on $\gamma$ occurs when $\phi(z)=$ $i / \pi z$ and $\phi_{1}=0$. We remark that Coifman and Meyer first showed in [4] how the $L_{p}$-boundedness of the singular Cauchy integral operator on $\gamma$ could be used to prove the $L_{p}$-boundedness of other convolution singular integral operators on $\gamma$.

In this paper we present a higher-dimensional analogue of this result, concerning the $L_{p}$-boundedness of convolution singular integral operators on a Lipschitz surface

$$
\Sigma=\left\{g(\mathbf{x}) e_{0}+\mathbf{x} \in \mathbb{R}^{n+1}: \mathbf{x} \in \mathbb{R}^{n}\right\}
$$

where $g$ is a Lipschitz function which satisfies $\|\nabla g\|_{\infty} \leq \tan \omega<\infty$. Here we have embedded $\mathbb{R}^{n+1}$ in the Clifford algebra $\mathbb{R}_{(n)}$ with identity $e_{0}$, and are considering convolution with right-monogenic (right Clifford-regular) functions $\phi$ which satisfy $|\phi(x)| \leq C|x|^{-n}$ on a sector

$$
S_{\mu}^{o}=\left\{x=x_{0}+\mathbf{x} \in \mathbb{R}^{n+1}:\left|x_{0}\right|<|\mathbf{x}| \tan \mu\right\}
$$

where $\mu>\omega$. Provided there exists an $L_{\infty}$ function $\underline{\phi}$ satisfying

$$
\underline{\phi}(R)-\underline{\phi}(r)=\int_{\substack{r<|x|<R \\ x \in \mathbb{R}^{n}}} \phi(x) d x,
$$

then the related convolution singular integral operator $T_{(\phi, \phi)}$ is bounded on $L_{p}(\Sigma)$ for $1<p<\infty$. A detailed statement is given in $\xi \overline{3}$, while the proof occupies $\S \S 4-6$.

The operators $T_{(\phi, \phi)}$ act on functions defined on the Lipschitz surface $\Sigma$ taking values in the Clifford algebra $\mathbb{R}_{(n)}$, its complexification $\mathbb{C}_{(n)}$, or indeed in any finite-dimensional left-Clifford module, such as those which are considered in the papers of Gilbert and Murray, e.g., [9].

As in the holomorphic case, we first derive quadratic estimates for those $L_{2}$ functions on $\Sigma$ which are in the Hardy spaces $H_{ \pm}^{2}(\Sigma)$ consisting of boundary 
values of left-monogenic functions defined on one side of the surface $\Sigma$ or the other. We then use the idea in [5] to find $L_{p}$ bounds for convolution with functions $\phi_{ \pm}$which are right-monogenic, not just on $S_{\mu}^{o}$, but also above or below $S_{\mu}^{o}$. Finally, we obtain the $L_{p}$ bounds for convolution with $\phi$ by decomposing $\phi$ as a sum of such functions $\phi_{+}$and $\phi_{-}$. As in the onedimensional case, the surprising thing is that quadratic estimates in the Hardy spaces $H_{ \pm}^{2}(\Sigma)$ lead to bounds on all of $L_{2}(\Sigma)$.

It should be possible to prove our theorem by other means, as for example by using the Clifford $\mathrm{Tb}$ theorem [7]. We remark that Clifford martingales have recently been developed by Gaudry, Long, and Qian and used to give a new proof of the Clifford Tb theorem [8].

The most important special case occurs when $\phi(x)=\frac{2}{\sigma_{n}} \frac{\bar{x}}{|x|^{n+1}}, \underline{\phi}=0$, and $T_{(\phi, \phi)}=C_{\Sigma}$, the singular Cauchy integral operator on $\Sigma$. This operator appears to have been first studied by Iftimie who proved in 1965 that it is a bounded operator on Hölder continuous functions on Lyapunov surfaces [10]. Although the $L_{p}$-boundedness of this operator on Lipschitz surfaces follows from the results in [3] using the Calderón rotation method, it is of interest to obtain such bounds directly using Clifford generalizations of the one-dimensional proofs. This was done by Murray for surfaces with small Lipschitz constants [17], though this restriction was removed in [13].

Observe that the singular double-layer potential operator on $\Sigma$ is the scalar part of the singular Cauchy integral operator on $\Sigma$.

Although the operators $T_{(\phi, \underline{ }}$ of Theorem 1.1 form an algebra, this is not true in higher dimensions for two reasons. First, it is not natural to define the convolution product $\phi_{1} * \phi_{2}$ of two right-monogenic functions $\phi_{1}$ and $\phi_{2}$ on $S_{\mu}^{o}$ unless $\phi_{2}$ is also left-monogenic. So we need to restrict our attention to functions which are two-sided monogenic. Second, in order to decompose such $\phi$ as $\phi=\phi_{+}+\phi_{-}$with $\phi_{+}$and $\phi_{-}$two-sided monogenic, we need the extra condition $(*)$ stated in $\S 3$.

We show that the operators $T_{(\phi, \phi)}$ corresponding to two-sided monogenic functions $\phi$ satisfying condition $(*)$ do form an algebra. Moreover $T_{(\phi, \phi)}=$ $T_{\phi_{+}}+T_{\phi_{-}}$where $T_{\phi_{+}}$and $T_{\phi_{-}}$are bounded operators from $L_{p}(\Sigma)$ to $H_{ \pm}^{p}(\Sigma)$.

That these operators are precisely those arising from an $H_{\infty}$-functional calculus of the operator $\mathbf{D}_{\Sigma}$ introduced by Coifman and used in [17] and [13] will be shown in [12]. Indeed $T_{(\phi, \phi)}=b\left(\mathbf{D}_{\Sigma}\right)$ where $b$ is the Fourier transform of $(\phi, \underline{\phi})$, extended to a sector in $\mathbb{C}^{n}$.

We remark that if we decompose $\mathbf{D}_{\Sigma}$ as a sum of operators $\mathbf{D}_{\Sigma}^{+}$and $\mathbf{D}_{\Sigma}^{-}$ acting in the Hardy spaces $H_{ \pm}^{2}(\Sigma)$, then $\mp \mathbf{D}_{\Sigma}^{ \pm}$are the infinitesimal generators of the semigroups $u(x) \rightarrow u\left(x \pm t e_{0}\right)$.

This paper is actually closely related to the paper [12] being written jointly with Tao Qian by two of the authors. Although in the current paper we have avoided using the characterisations by Fourier transforms just mentioned, many of the statements and results in this paper were actually found by using those 
ideas. So we would especially like to express our appreciation to Qian with whom we have been developing this topic.

We are also indebted to the other mathematicians with whom we have discussed this material, in particular to John Ryan.

\section{Clifford ANALYSIS}

We embed the vector space $\mathbb{R}^{n+1}$ in the real $2^{n}$-dimensional Clifford algebra $\mathbb{R}_{(n)}$ or in the complex $2^{n}$-dimensional Clifford algebra $\mathbb{C}_{(n)}$ in the usual way. Let $e_{0}, e_{1}, e_{2}, \ldots, e_{n}$ be the standard basis of $\mathbb{R}^{n+1}$, and denote the basis vectors of $\mathbb{R}_{(n)}$ or $\mathbb{C}_{(n)}$ by $e_{S}$, where $S$ is any subset of $\{1,2, \ldots, n\}$. Make the identifications $e_{0}=e_{\phi}$ and $e_{j}=e_{\{j\}}$ for $1 \leq j \leq n$, and define the associative multiplication of basis vectors by

$$
\begin{aligned}
e_{0} & =1, \\
e_{j}^{2} & =-e_{0}=-1 \quad \text { for } 1 \leq j \leq n, \\
e_{j} e_{k} & =-e_{k} e_{j}=e_{\{j, k\}} \quad \text { for } 1 \leq j<k \leq n,
\end{aligned}
$$

and

$$
e_{j_{1}} e_{j_{2}} \cdots e_{j_{s}}=e_{S} \quad \text { if } 1 \leq j_{1}<j_{2}<\cdots<j_{s} \leq n \text { and } S=\left\{j_{1}, j_{2}, \ldots, j_{s}\right\} .
$$

The product of two elements $u=\sum_{S} u_{S} e_{S}$ and $v=\sum_{S} v_{S} e_{S}$ in $\mathbb{R}_{(n)}\left(\right.$ or $\mathbb{C}_{(n)}$ ) is $u v=\sum_{S, T} u_{S} v_{T} e_{S} e_{T}$. The term $u_{\phi} e_{\phi}$ is usually written as $u_{0} e_{0}$ or just $u_{0}$, and is called the scalar part of $u$.

We use the Euclidean norm $|u|$ in $\mathbb{R}_{(n)}$ (or $\mathbb{C}_{(n)}$ ), defined by $|u|^{2}=\sum_{S}\left|u_{S}\right|^{2}$ $=(u, u)$, and the inner product $(u, v)=\sum_{S} u_{S} \bar{v}_{S}=(\bar{v} u)_{0}$ where $\bar{v}_{S}$ is the complex conjugate of $v_{S}$ and $\bar{v}=\sum_{S} \bar{v}_{S} \bar{e}_{S}$ with $\bar{e}_{S}= \pm e_{S}$, the sign chosen so that $\bar{e}_{S} e_{S}=e_{S} \bar{e}_{S}=1$.

Let us now outline some analysis. The results we quote here can be found in [1]. The differential operator $D=\frac{\partial}{\partial x_{0}}+\mathbf{D}$ where $\mathbf{D}=\sum_{k=1}^{n} \frac{\partial}{\partial x_{k}} e_{k}$ acts on $C^{1}$-functions $f$ to give

$$
D f=\sum_{k=0}^{n} \frac{\partial f_{S}}{\partial x_{k}} e_{k} e_{S}
$$

and also

$$
f D=\sum_{k=0}^{n} \frac{\partial f_{S}}{\partial x_{k}} e_{S} e_{k}
$$

A $C^{1}$-function defined on an open subset of $\mathbb{R}^{n+1}$ with values in $\mathbb{R}_{(n)}$ or $\mathbb{C}_{(n)}$ is called left-monogenic if $D f=0$ and right-monogenic if $f D=0$. All analytic functions $F$ on domains in $\mathbb{R}^{n}$ have both left- and right-monogenic extensions to domains in $\mathbb{R}^{n+1}$. These two extensions coincide if and only if $\mathbf{D} F=F \mathbf{D}$, so in particular they coincide if $F$ is scalar valued. 
The most important functions which are both left- and right-monogenic are the Cauchy kernels

$$
\frac{1}{\sigma_{n}} \frac{\overline{y-x}}{|y-x|^{n+1}} \quad \text { for } x \in \mathbb{R}^{n+1} \backslash\{y\}
$$

where $\sigma_{n}$ is the volume of the unit n-sphere in $\mathbb{R}^{n+1}$.

The following version of Cauchy's theorem and the Cauchy integral formula holds.

Theorem 2.1. Let $\Omega$ be a bounded open subset of $\mathbb{R}^{n+1}$ with Lipschitz boundary $\partial \Omega$ and exterior unit normal $n(y)$ defined for almost all $y \in \partial \Omega$. Suppose $f$ is left-monogenic and $g$ is right-monogenic on a neighbourhood of $\Omega^{-}=\Omega \cup \partial \Omega$. Then

$$
\begin{gathered}
\int_{\partial \Omega} g(y) n(y) f(y) d S_{y}=0, \\
\frac{1}{\sigma_{n}} \int_{\partial \Omega} g(y) n(y) \frac{\overline{y-x}}{|y-x|^{n+1}} d S_{y}=\left\{\begin{aligned}
g(x), & x \in \Omega^{-} \\
0, & x \notin \Omega^{-},
\end{aligned}\right. \\
\frac{1}{\sigma_{n}} \int_{\partial \Omega} \frac{\overline{y-x}}{|y-x|^{n+1}} n(y) f(y) d S_{y}=\left\{\begin{aligned}
f(x), & x \in \Omega^{-}, \\
0, & x \notin \Omega^{-} .
\end{aligned}\right.
\end{gathered}
$$

A result we need is the following.

Theorem 2.2. Suppose $f$ is right-monogenic on $\mathbb{R}^{n+1} \backslash\{0\}$ and satisfies the bound $|f(x)| \leq C /|x|^{n}$ for $x \in \mathbb{R}^{n+1} \backslash\{0\}$. Then $f(x)=c \bar{x} /|x|^{n+1}$ for some constant $c \in \mathbb{C}_{(n)}$.

The proof can be found in $\S 12$, Chapter 2 of [1].

We note that parts (i) and (iii) of Theorem 2.1 remain valid when $f$ is a leftmonogenic function taking its values in $\mathscr{X}$, where $\mathscr{X}$ is a finite-dimensional left Clifford module. That is, $\mathscr{X}$ is a finite-dimensional real (or complex) linear space together with a representation of $\mathbb{R}_{(n)}\left(\right.$ or $\left.\mathbb{C}_{(n)}\right)$ as linear operators on $\mathscr{X}$. If $u \in \mathbb{R}_{(n)}\left(\right.$ or $\left.\mathbb{C}_{(n)}\right)$ and $v \in \mathscr{X}$, we denote the action of $u$ on $v$ by $u v$.

We consider $\mathscr{X}$ together with a norm $\|\cdot\|$ and note that there exists a constant $C$ such that $\|u v\| \leq C|u|\|v\|$ for all $u \in \mathbb{R}_{(n)}$ (or $\mathbb{C}_{(n)}$ ) and $v \in \mathscr{X}$. However we do not equip $\mathscr{X}$ with an inner product, and in particular do not require that the basis vectors $e_{j}$ be represented by skew-adjoint operators.

\section{CONVOLUTION SINGULAR INTEGRALS ON LIPSCHITZ SURFACES}

Throughout this paper $\Sigma$ denotes the Lipschitz surface consisting of points $x=g(\mathbf{x})+\mathbf{x}=g(\mathbf{x}) e_{0}+\mathbf{x} \in \mathbb{R}^{n+1}$, where $\mathbf{x} \in \mathbb{R}^{n}$, and $g$ is a real-valued Lipschitz function which satisfies $\|\nabla g\|_{\infty} \leq \tan \omega<\infty$ where $0 \leq \omega<\pi / 2$.

Moreover $\mathscr{X}$ denotes a finite-dimensional left module over the Clifford algebra $\mathbb{R}_{(n)}\left(\right.$ or $\left.\mathbb{C}_{(n)}\right)$. If $1 \leq p<\infty$ then $L_{p}(\Sigma)=L_{p}(\Sigma, \mathscr{X})$ is the space of 
equivalence classes of functions $u: \Sigma \rightarrow \mathscr{X}$ which are measurable with respect to $d S_{x}=\sqrt{1+|\nabla g(x)|^{2}} d x$, and for which

$$
\|u\|_{p}=\left\{\int_{\Sigma}\|u(x)\|^{p} d S_{x}\right\}^{1 / p}<\infty, \quad 1 \leq p<\infty .
$$

Our aim is to prove the $L_{p}$-boundedness of convolution singular integral operators associated with right-monogenic functions on open sectors $S_{\mu}^{o}$ in $\mathbb{R}^{n+1}$ defined by

$$
S_{\mu}^{o}=\left\{x=x_{0}+\mathbf{x} \in \mathbb{R}^{n+1}:\left|x_{0}\right|<|\mathbf{x}| \tan \mu\right\}
$$

when $\omega<\mu<\pi / 2$.

Define $K\left(S_{\mu}^{o}\right)$ to be the Banach space of pairs $(\phi, \underline{\phi})$ of functions with $\phi$ right-monogenic from $S_{\mu}^{o}$ to $\mathbb{R}_{(n)}$ (or $\mathbb{C}_{(n)}$ ) and with $\underline{\phi}$ a differentiable function on $(0, \infty)$ such that $\underline{\phi}^{\prime}(r)=\int_{|x|=r} \phi(x) d x$ (where the integral is over the $(n-1)$-sphere $S(0, r)$ in $\left.\mathbb{R}^{n}\right)$ and

$$
\|(\phi, \underline{\phi})\|_{K\left(S_{\mu}^{o}\right)}=\frac{\sigma_{n}}{2} \sup \left\{|x|^{n}|\phi(x)|: x \in S_{\mu}^{o}\right\}+\sup \{|\underline{\phi}(r)|: r>0\}<\infty
$$

where $\sigma_{n}$ is the volume of the unit $n$-sphere in $\mathbb{R}^{n+1}$.

Note that $\underline{\phi}$ is determined by $\phi$ up to an additive constant, and that

$$
\underline{\phi}(R)-\underline{\phi}(r)=\int_{\substack{r<|x|<R \\ x \in \mathbb{R}^{n}}} \phi(x) d x
$$

when $0<r<R$.

Given $(\phi, \underline{\phi}) \in K\left(S_{\mu}^{o}\right)$ we extend $\underline{\phi}$ to the open cone

$$
T_{\mu}^{o}=\left\{y=y_{0}+\mathbf{y} \in \mathbb{R}^{n+1}: y_{0}>|\mathbf{y}| \cot \mu\right\}
$$

by

$$
\underline{\phi}(r t)=\underline{\phi}(r)-\int_{A(r, t)}(\phi(x)+\phi(-x)) n(x) d S_{x}, \quad t \in T_{\mu}^{o},|t|=1, r>0,
$$

where $A(r, t)=\left\{x \in \mathbb{R}^{n+1}:|x|=r, x_{0}>0,\langle x, t\rangle<0\right\}$ and $n(x)$ is the exterior normal to $A(r, t)$ at the point $x$. It is easy to see that

$$
\sup \left\{|\underline{\phi}(x)|: x \in T_{\mu}^{o}\right\} \leq\|(\phi, \underline{\phi})\|_{K\left(S_{\mu}^{o}\right)} .
$$

Theorem 3.1. Suppose that $\omega<\mu<\pi / 2$ and $1<p<\infty$. Given $(\phi, \underline{\phi}) \in$ $K\left(S_{\mu}^{o}\right)$, there is a bounded linear operator $T_{(\phi, \phi)}$ on $L_{p}(\Sigma)$ defined for all $u \in$ $L_{p}(\Sigma)$ and almost all $x \in \Sigma$ by

$$
\left(T_{(\phi, \underline{\phi})} u\right)(x)=\lim _{\varepsilon \rightarrow 0+}\left\{\int_{|x-y| \geq \varepsilon} \phi(x-y) n(y) u(y) d S_{y}+\underline{\phi}(\varepsilon n(x)) u(x)\right\} .
$$

Moreover

$$
\left\|T_{(\phi, \underline{\phi})} u\right\|_{p} \leq C_{\omega, \mu, p}\|(\phi, \underline{\phi})\|_{K\left(S_{\mu}^{o}\right)}\|u\|_{p}
$$


for some constant $C_{\omega, \mu, p}$ which depends only on $\omega, \mu$ and $p$ (and dimension $n)$.

In particular, when $(\phi, \underline{\phi})=(2 k, 0)$ where $k(x)=\frac{1}{\sigma_{n}} \frac{\bar{x}}{|x|^{n+1}}$, then $T_{(\phi, \underline{\phi})}=$ $C_{\Sigma}$, the Cauchy singular integral operator on $\Sigma$ given by

$$
C_{\Sigma} u(x)=\text { p.v. } \frac{2}{\sigma_{n}} \int_{\Sigma} \frac{\overline{x-y}}{|x-y|^{n+1}} n(y) u(y) d S_{y}
$$

and we have the known result that $C_{\Sigma}$ is bounded on $L_{p}(\Sigma)$ for $1<p<\infty$.

More generally, if $\phi(x)$ is an odd function which is right-monogenic and satisfies $|\phi(x)| \leq C|x|^{-n}$ for all $x \in S_{\mu}^{o}$, then the principal value convolution operator $\phi * u$ equals $T_{(\phi, 0)}$ and is therefore bounded on $L_{p}(\Sigma)$.

We postpone the proof until $\S 6$, though a special case, which implies the boundedness of the Cauchy singular integral operator, will be proved in $\S 5$.

The space $K\left(S_{\mu}^{o}\right)$ is not a convolution algebra. But if we define

$$
M\left(S_{\mu}^{o}\right)=\left\{(\phi, \underline{\phi}) \in K\left(S_{\mu}^{o}\right): \quad \phi \text { is left-monogenic and }(*) \text { holds }\right\}
$$

where

$$
\text { (*) } \int_{\substack{|y|=r \\ y \in \mathbb{R}^{n}}}\langle y, x\rangle(\phi(y) n(y)-n(y) \phi(y)) d S_{y}-(\underline{\phi}(r) x-x \underline{\phi}(r))=0,
$$

then $M\left(S_{\omega}\right)=\bigcup_{\mu>\omega} M\left(S_{\mu}^{o}\right)$ is a convolution algebra. Details will be given in $\S 6$, where we prove the following result.

Theorem 3.2. Suppose $1<p<\infty$. If $(\phi, \underline{\phi}),(\psi, \underline{\psi}) \in M\left(S_{\omega}\right)$, then

$$
T_{(\phi, \underline{\phi})} \cdot T_{(\psi, \underline{\psi})}=T_{(\phi, \underline{\phi}) *(\psi, \underline{\psi})} \cdot
$$

The algebra of oprators $\left\{T_{(\phi, \underline{\phi})}:(\phi, \underline{\phi}) \in M\left(S_{\omega}\right)\right\}$ is precisely that corresponding to the $H_{\infty}$-functional calculus of $\mathbf{D}_{\Sigma}$ developed in [12].

\section{REVERSE SQUARE FUNCTION ESTIMATES}

Let $\Omega_{+}$be the domain in $\mathbb{R}^{n+1}$ above the surface $\Sigma$ and $\Omega_{-}$the one below. Suppose $\sigma(x)=\operatorname{dist}(x, \Sigma)$. Then $\mathscr{H}_{+}=L_{2}\left(\Omega_{+}, \mathbb{C}_{(n)}, \sigma(x) \bar{d} x\right)$ is a Hilbert space with norm

$$
\begin{aligned}
\|f\|_{\mathscr{H}_{+}} & =\left(\iint_{\Omega_{+}}|f(x)|^{2} \sigma(x) d x\right)^{1 / 2} \\
& =\sup _{g \in B}\left|\iint_{\Omega_{+}}(f(x), g(x)) \sigma(x) d x\right|
\end{aligned}
$$

where

$$
B=\left\{f \in \mathscr{H}_{+}:\|f\|_{\mathscr{H}_{+}} \leq 1, f \text { compactly supported in } \Omega_{+}\right\} .
$$

Similarly we define $\mathscr{H}_{-}$. 
Theorem 4.1 (reverse square function estimate). Suppose that $f: \Omega_{+} \rightarrow \mathbb{C}_{(n)}$ is right-monogenic and continuous to the boundary $\Sigma$. If $|f(x)| \leq c /(1+|x|)^{n}$ and $|\nabla f(x)| \leq c /(1+|x|)^{n+1}$ for all $x \in \Omega_{+} \cup \Sigma$, then

$$
\left\{\int_{\Sigma}|f(x)|^{2} d S_{x}\right\}^{1 / 2} \leq C\|\nabla f\|_{\mathscr{P}_{+}}
$$

where $\|\nabla f\|_{\mathscr{H}_{+}}^{2}=\sum_{j=0}^{n}\left\|\frac{\partial}{\partial x_{j}} f\right\|_{\mathscr{H}_{+}}^{2}$.

It is easy to see that $f$ is the Cauchy integral of its restriction to $\Sigma$ and $\left\|f\left(x+t e_{0}\right)\right\|_{L_{2}(\Sigma, d x)} \rightarrow 0$ as $t \rightarrow \infty$. So

$$
\begin{aligned}
\int_{\Sigma}|f(x)|^{2} d S_{x} & =\int_{0}^{\infty} \int_{\Sigma} \frac{\partial}{\partial t}\left|f\left(x+t e_{0}\right)\right|^{2} d S_{x} d t \\
& =\int_{0}^{\infty} \int_{\Sigma} \frac{\partial}{\partial t}\left(f\left(x+t e_{0}\right), f\left(x+t e_{0}\right)\right) d S_{x} d t \\
& =2 \mathscr{R} e \iint_{\Omega_{+}}\left(\left(\frac{\partial}{\partial x_{0}} f\right)(x), f(x)\right) d x .
\end{aligned}
$$

For functions $f$ and $g$ defined on $\Omega_{+}$, set the bilinear form

$$
B(f, g)=\iint_{\Omega_{+}}\left(\left(\frac{\partial}{\partial x_{0}} f\right)(x), g(x)\right) d x .
$$

The following result was first proved by Dahlberg, who only required that $f$ be harmonic. The extra assumption of monogenicity will allow us to give a simpler proof, although some of the ingredients are the same as in Dahlberg's proof [6].

For a function $g$ on $\Omega_{+}$, the nontangential maximal function is

$$
N(g)(x)=\sup _{y \in x+\Gamma(\theta)}|g(y)|, \quad x \in \Sigma,
$$

where $\Gamma(\theta)=\left\{x=x_{0}+\mathbf{x}:|\mathbf{x}|<x_{0} \tan \theta\right\}$ for some $\theta \in\left(0, \frac{\pi}{2}-\omega\right)$.

Lemma 4.2. Suppose $f$ satisfies the conditions in Thoerem 4.1, $g$ is smooth on $\Omega_{+},|g(x)| \leq c /(1+|x|)^{n},|\nabla g(x)| \leq c /(1+|x|)^{n+1}$. Then

$$
|B(f, g)| \leq C\left(\iint_{\Omega_{+}}|\nabla f|^{2} \sigma(x) d x\right)^{1 / 2}|\|g\||
$$

where

$$
|\|g\||=\left(\iint_{\Omega_{+}}|\nabla g|^{2} \sigma(x) d x\right)^{1 / 2}+\left(\int_{\Sigma} N(g)^{2} d S_{x}\right)^{1 / 2}
$$

and $N(g)$ is the nontangential maximal function of $g$.

Before proving Lemma 4.2, let us indicate how it implies Theorem 4.1. We have already got

$$
\int_{\Sigma}|f(x)|^{2} d S_{x}=2 \mathscr{R} e B(f, f) \leq C\left(\iint_{\Omega_{+}}|\nabla f|^{2} \sigma(x) d x\right)^{1 / 2}|\|f\|| .
$$


We claim that

$$
\int_{\Sigma}|N(f)(x)|^{2} d S_{x} \leq C \int_{\Sigma}|f(x)|^{2} d S_{x} .
$$

Indeed, let $\mathscr{C}_{\Sigma}(f)$ denote the Cauchy integral of the boundary values of $f$. That is,

$$
\mathscr{C}_{\Sigma}(f)(x)= \pm \int_{\Sigma} k(x-y) n(y) f(y) d S_{y}, \quad x \in \Omega_{ \pm} .
$$

Then by using Cauchy's integral formula and the assumption that $f$ decays at $\infty$ we get $\mathscr{C}_{\Sigma}(f)=f$ on $\Omega_{+}, \mathscr{C}_{\Sigma}(f)=0$ on $\Omega_{-}$. If $x \in \Omega_{+}$, we can find $y(x) \in \Omega_{-}$such that $|x-y(x)| \approx \operatorname{dist}(x, \Sigma) \approx \operatorname{dist}(y(x), \Sigma)$. By direct computation on the Cauchy kernel, the nontangential maximal function of $f(x)=\mathscr{C}_{\Sigma}(f)(x)-\mathscr{C}_{\Sigma}(f)(y(x))$ is controlled by the Hardy-Littlewood maximal function $M(f)$ of $f$ pointwise on $\Sigma$. So

$$
\int_{\Sigma} N(f)(x)^{2} d S_{x} \leq C \int_{\Sigma} M(f)(x)^{2} d S_{x} \leq C \int_{\Sigma}|f(x)|^{2} d S_{x}
$$

Thus we get

$$
\begin{aligned}
\int_{\Sigma}|f(x)|^{2} d S_{x} \leq & C\left(\iint_{\Omega_{+}}|\nabla f|^{2} \sigma(x) d x\right)^{1 / 2} \\
& \times\left\{C\left(\int_{\Sigma}|f(x)|^{2} d S_{x}\right)^{1 / 2}+\left(\iint_{\Omega_{+}}|\nabla f|^{2} \sigma(x) d x\right)^{1 / 2}\right\} .
\end{aligned}
$$

Using $A^{2} \leq C^{2} B^{2}+C A B \Rightarrow A \leq 2 C B$, we get

$$
\int_{\Sigma}|f(x)|^{2} d S_{x} \leq C \iint_{\Omega_{+}}|\nabla f|^{2} \sigma(x) d x
$$

as desired.

Now we go back to prove Lemma 4.2. Following Dahlberg, we use a good bilipschitz change of variables. Choose $\phi$ to be a smooth function on $\mathbb{R}^{n}$, compactly supported, $\int_{\mathbb{R}^{n}} \phi(x) d x=1$, and define $\phi_{\delta}(x)=\delta^{-n} \phi\left(\frac{x}{\delta}\right)$,

$$
\rho(x)=x_{0}+\mathbf{x}+\phi_{x_{0}} * g(\mathbf{x}), \quad x=\mathbf{x}+x_{0} \in \mathbb{R}_{+}^{n+1} \text {. }
$$

For properly chosen $\phi, \rho$ defines a bilipschitz map from $\mathbb{R}_{+}^{n+1}$ onto $\Omega_{+}$such that $|\nabla \rho|^{2} x_{0} d x$ is a Carleson measure. (The existence of such a change of variables is due to Dahlberg, but this particularly simple mapping was found by Kenig and Stein.)

After making the change of variables, we can rewrite $B(f, g)$ as

$$
\iint_{\mathbb{R}_{+}^{n+1}}\left(\sum_{i=1}^{n} a_{i}(x) \frac{\partial}{\partial x_{i}} F(x), G(x)\right) d x \quad F=f \cdot \rho, G=g \cdot \rho .
$$

The $a_{i} \in L_{\infty}\left(\mathbb{R}_{+}^{n+1}, \mathbb{R}_{(n)}\right)$ and $\left|\nabla a_{i}(x)\right|^{2} x_{0} d x$ are Carleson measures on $\mathbb{R}_{+}^{n+1}$. We have used the monogenicity of $f$ to replace the $\partial / \partial x_{0}$ term by terms of $\partial / \partial x_{j}, j=1,2, \ldots, n$, with coefficients satisfying the above conditions. 
Let $R_{j}$ denote the Riesz transforms and $\Delta$ the negative of the Laplacian on $\mathbb{R}^{n}$. Then the above becomes

$$
\begin{aligned}
B(f, g) & =\iint_{\mathbb{R}_{+}^{n+1}}\left(\sum_{i=1}^{n}\left(\Delta^{1 / 4} R_{i} F\right)(x), \Delta^{1 / 4}\left(\bar{a}_{i} G\right)(x)\right) d x \\
& \leq C\left(\iint_{\mathbb{R}_{+}^{n+1}}\left|\Delta^{1 / 4} F(x)\right|^{2} d x\right)^{1 / 2}\left(\sum_{i=1}^{n} \iint_{\mathbb{R}_{+}^{n+1}}\left|\Delta^{1 / 4}\left(\bar{a}_{i} G\right)(x)\right|^{2} d x\right)^{1 / 2} .
\end{aligned}
$$

Lemma 4.3. If $H$ is a smooth function defined on $\mathbb{R}_{+}^{n+1}$ and $H, \nabla H \in L_{2}\left(\mathbb{R}_{+}^{n+1}\right)$, then

$$
\int_{\mathbb{R}^{n}}\left|\Delta^{1 / 4} H\right|^{2} d x \leq C \iint_{\mathbb{R}_{+}^{n+1}}|\nabla H|^{2} d x .
$$

This well-known result can be derived by applying the even better known trace estimate

$$
\int_{\mathbb{R}^{n}}\left|\Delta^{1 / 4} H_{t}\right|^{2} d x \leq C \iint_{\mathbb{R}_{+}^{n+1}}\left(\left|\nabla H_{t}\right|^{2}+\left|H_{t}\right|^{2}\right) d x
$$

to the function $H_{t}(x)=H(t x)$, and letting $t \rightarrow \infty$.

From the lemma we get that

$$
\begin{aligned}
\int_{0}^{\infty} \int_{\mathbb{R}^{n}}\left|\Delta^{1 / 4} F\left(x_{0}+\mathbf{x}\right)\right|^{2} d \mathbf{x} d x_{0} & \leq C \int_{0}^{\infty} \int_{\mathbb{R}^{n}} \int_{0}^{\infty}\left|\nabla F\left(x_{0}+\mathbf{x}+t e_{0}\right)\right|^{2} d t d \mathbf{x} d x_{0} \\
& =C \int_{0}^{\infty} \int_{\mathbb{R}^{n}}\left|\nabla F\left(\mathbf{x}+t e_{0}\right)\right|^{2} t d t d \mathbf{x} \\
& \leq C \iint_{\Omega_{+}}|\nabla f|^{2} \sigma(x) d x .
\end{aligned}
$$

Similarly

$$
\begin{aligned}
\iint_{\mathbb{R}_{+}^{n+1}}\left|\Delta^{1 / 4}\left(\bar{a}_{i} G\right)(x)\right|^{2} d x & \leq C \int_{0}^{\infty} \int_{\mathbb{R}^{n}}\left|\nabla\left(\bar{a}_{i} G\right)\left(\mathbf{x}+t e_{0}\right)\right|^{2} t d \mathbf{x} d t \\
& \leq C|\|G\||^{2} \leq C|\|g\||^{2}
\end{aligned}
$$

because $\bar{a}_{i} \in L_{\infty}\left(\mathbb{R}_{+}^{n+1}, \mathbb{R}_{(n)}\right)$ and $\left|\nabla \bar{a}_{i}(x)\right|^{2} x_{0} d x$ are Carleson measures. This proves Lemma 4.2 and therefore Theorem 4.1.

\section{OPERATOR BOUNDS}

Let us first prove the special case of Theorem 3.1 where $\phi$ can be extended monogenically to $\mathbb{R}_{+}^{n+1}$ or $\mathbb{R}_{-}^{n+1}$. More precisely, for $0<\mu<\pi / 2$, define the open cones $C_{\mu+}^{o}, C_{\mu-}^{o}$ in $\mathbb{R}^{n+1}$ by

$$
C_{\mu+}^{o}=\left\{x=x_{0}+\mathbf{x} \in \mathbb{R}^{n+1}: x_{0}>-|\mathbf{x}| \tan \mu\right\}, \quad C_{\mu-}^{o}=-C_{\mu+}^{o} .
$$

Then $S_{\mu}^{o}=C_{\mu+}^{o} \cap C_{\mu-}^{o}$. 
Define the Banach spaces $K\left(C_{\mu \pm}^{o}\right)$ to be the spaces of right-monogenic functions $\phi$ on $C_{\mu \pm}^{o}$ for which

$$
\|\phi\|_{K\left(C_{\mu \pm}^{o}\right)}=\frac{\sigma_{n}}{2} \sup \left\{|x|^{n}|\phi(x)|: x \in C_{\mu \pm}^{o}\right\}<\infty .
$$

Given $\phi \in K\left(C_{\mu \pm}^{o}\right)$ and $r>0$, define

$$
\underline{\phi}_{ \pm}(r)=\int_{\substack{|x|=r \\ \pm x_{0}>0}} \phi(x) n(x) d S_{x}
$$

where $n(x)$ is the upward pointing normal to the sphere $\left\{x \in \mathbb{R}^{n+1}:|x|=r\right\}$.

In $\S 6$ we will show that $K\left(S_{\mu}^{o}\right) \cong K\left(C_{\mu+}^{o}\right) \oplus K\left(C_{\mu-}^{o}\right)$ and use this decomposition to prove that Theorem 3.1 is a consequence of the following result.

Theorem 5.1. Suppose that $\omega<\mu<\pi / 2$ and $1<p<\infty$. Given $\phi \in K\left(C_{\mu+}^{o}\right)$ or $K\left(C_{\mu-}^{o}\right)$, there is a bounded linear operator $T_{\phi}$ on $L_{p}(\Sigma)$ defined for all $u \in L_{p}(\Sigma)$ and almost all $x \in \Sigma$ by

$$
\begin{aligned}
& \left(T_{\phi} u\right)(x)=\lim _{\delta \rightarrow 0+}\left\{\int_{\Sigma} \phi(x \pm \delta-y) n(y) u(y) d S_{y}\right\} \\
& =\lim _{\varepsilon \rightarrow 0+}\left\{\int_{|x-y| \geq \varepsilon} \phi(x-y) n(y) u(y) d S_{y}+\underline{\phi}_{ \pm}(\varepsilon n(x)) u(x)\right\}
\end{aligned}
$$

where $n(x)$ is the upward pointing unit normal vector to $\Sigma$ which is defined at almost all $x \in \Sigma$. Moreover

$$
\left\|T_{\phi} u\right\|_{p} \leq C_{\omega, \mu, p}\|\phi\|_{K\left(C_{\mu \pm}^{o}\right)}\|u\|_{p}
$$

for some constants $C_{\omega, \mu, p}$ which depend only on $\omega, \mu$ and $p$.

The following known results follow directly from this theorem.

Corollary 5.2. Let $k(x)=\frac{1}{\sigma_{n}} \frac{\bar{x}}{|x|^{n+1}}$ when $x \neq 0$. Then there are bounded linear Cauchy operators $P_{+}, P_{-}$and $C_{\Sigma}$ on $L_{p}(\Sigma)$ defined for all $u \in L_{p}(\Sigma)$ and almost all $x \in \Sigma$ by

$$
\left(P_{ \pm} u\right)(x)= \pm \lim _{\delta \rightarrow 0^{+}} \int_{\Sigma} k(x \pm \delta-y) n(y) u(y) d S_{y}
$$

and

$$
\left(C_{\Sigma} u\right)(x)=2 \text { p.v. } \int_{\Sigma} k(x-y) n(y) u(y) d S_{y} .
$$

Moreover, $P_{ \pm}=\frac{1}{2}\left( \pm C_{\Sigma}+I\right)= \pm T_{k_{ \pm}}$where $k_{ \pm}=\left.k\right|_{C_{\mu \pm}^{o}}$. (Note that $\underline{k}_{ \pm}=$ $\pm \frac{1}{2}$.) So $P_{+}+P_{-}=I$ and $P_{+}-P_{-}=C_{\Sigma}$.

These are Plemelj formulae [10].

Proof of Theorem 5.1. We prove the case when $\phi \in K\left(C_{\mu+}^{o}\right)$. Our proof is an adaptation of the first proof in the paper [5] by Coifman, Jones, and Semmes on the $L_{p}$-boundedness of the Cauchy singular integral operator on a Lipschitz curve. It depends on the reverse square function estimates presented in Theorem 4.1 and the following lemma. 
Lemma 5.3. Suppose $f$ is a function in $\mathscr{H}_{+}$with compact support in $\Omega_{+}, \phi \in$ $K\left(C_{\mu+}^{o}\right), j=0,1, \ldots, n$, and $\delta>0$. Define

$$
S_{\phi, \delta, j} f(y)=\iint_{\Omega_{+}} f(x) \frac{\partial \phi}{\partial x_{j}}(x-y+\delta) \sigma(x) d x, \quad y \in \bar{\Omega}_{-} .
$$

Then

$$
\left\|S_{\phi, \delta, j} f\right\|_{L_{2}(\Sigma)} \leq C\|\phi\|_{K\left(C_{\mu+}^{o}\right)}\|f\|_{\mathscr{H}_{+}}
$$

where $C$ depends only on $\mu$ and $\omega$.

Proof. Since $f$ is compactly supported we have $\left|S_{\phi, \delta, j} f(x)\right| \leq c /(1+|x|)^{n}$ and $\left|\nabla S_{\phi, \delta, j} f(x)\right| \leq c /(1+|x|)^{n+1}$. Since $\phi$ is right-monogenic on $C_{\mu+}^{o}, S_{\phi, \delta, j} f$ is right-monogenic on $\bar{\Omega}_{-}$. By Theorem 4.1, we have

$$
\left\|S_{\phi, \delta, j} f\right\|_{L_{2}(\Sigma)} \leq C\left\|\nabla\left(S_{\phi, \delta, j} f\right)\right\|_{\mathscr{H}_{-}} .
$$

Since

$$
\left|\nabla_{y} \frac{\partial \phi}{\partial x_{j}}(x-y)\right| \leq \frac{C\|\phi\|_{K\left(C_{\mu+}^{o}\right)}}{|x-y|^{n+2}} / \sin ^{n+2}\left(\frac{\mu-\omega}{2}\right) \quad \forall x-y \in C_{(\mu+\omega) / 2}^{o},
$$

we have

$$
\begin{aligned}
\left|\nabla\left(S_{\phi, \delta, j} f\right)(y)\right| & =\left|\iint_{\Omega_{+}} f(x) \nabla_{y} \frac{\partial \phi}{\partial x_{j}}(x-y+\delta) \sigma(x) d x\right| \\
& \leq C\|\phi\|_{K\left(C_{\mu+}^{o}\right)} \iint_{\Omega_{+}} \frac{|f(x)| \sigma(x)}{|x-y+\delta|^{n+2}} d x
\end{aligned}
$$

where $C$ depends on $\mu$ and $\omega$.

Since

$$
\sup _{y \in \Omega_{-}} \iint_{\Omega_{+}} \frac{\sigma(y)^{1 / 2} \sigma(x)^{1 / 2}}{|x-y+\delta|^{n+2}} d x \leq C
$$

and

$$
\sup _{x \in \Omega_{+}} \iint_{\Omega_{-}} \frac{\sigma(y)^{1 / 2} \sigma(x)^{1 / 2}}{|x-y+\delta|^{n+2}} d y \leq C
$$

with $C$ independent of $\delta$ (notice that $\sigma(x) \leq|x-y|, \sigma(y) \leq|x-y|$ for $x \in \Omega_{+}, y \in \Omega_{-}$), by Schur's lemma,

$$
\left\|\iint_{\Omega_{+}} \frac{|f(x)| \sigma(x)}{|x-y+\delta|^{n+2}} d x\right\|_{\mathscr{L}_{-}} \leq C\|f\|_{\mathscr{H}_{+}} .
$$

Therefore

$$
\left\|S_{\phi, \delta, j} f\right\|_{L_{2}(\Sigma)} \leq C\left\|\nabla\left(S_{\phi, \delta, j} f\right)\right\|_{\mathscr{H}_{-}} \leq C\|\phi\|_{K\left(C_{\mu+}^{o}\right)}\|f\|_{\mathscr{K}_{+}} .
$$

This proves the lemma. 
Now we are almost ready to prove Theorem 5.1. Consider first scalar-valued functions $u \in L_{p}(\Sigma), 1<p<\infty$. Then, for $\delta>0, W_{\phi_{\delta}} u$ is right-monogenic on $\bar{\Omega}_{+}$where

$$
\left(W_{\phi_{\delta}} u\right)(x)=\int_{\Sigma} \phi(x+\delta-y) u(y) d S_{y}, \quad x \in \bar{\Omega}_{+} .
$$

Let us first consider $u \in L_{2}(\Sigma)$ which is compactly supported on $\Sigma$, in which case it is clear that $W_{\phi_{\delta}} u$ satisfies Theorem 4.1. Thus by applying it, we have

$$
\begin{aligned}
\left\|W_{\phi_{\delta}} u\right\|_{L_{2}(\Sigma)} & \leq C\left\|\nabla\left(W_{\phi_{\delta}} u\right)\right\|_{\mathscr{C}_{+}} \\
& \leq C \sum_{j=0}^{n} \sup _{f_{j} \in B}\left|\iint_{\Omega_{+}}\left(\frac{\partial}{\partial x_{j}}\left(W_{\phi_{\delta}} u\right)(x), \bar{f}_{j}(x)\right) \sigma(x) d x\right| .
\end{aligned}
$$

But

$$
\begin{aligned}
\mid \iint_{\Omega_{+}} & \left(\frac{\partial}{\partial x_{j}}\left(W_{\phi_{\delta}} u\right)(x), \bar{f}_{j}(x)\right) \sigma(x) d x \mid \\
= & \left|\iint_{\Omega_{+}} \int_{\Sigma}\left(\frac{\partial \phi}{\partial x_{j}}(x+\delta-y) u(y), \bar{f}_{j}(x)\right) d S_{y} \sigma(x) d x\right| \\
= & \left|\int_{\Sigma} \iint_{\Omega_{+}}\left(f_{j}(x) \frac{\partial \phi}{\partial x_{j}}(x+\delta-y)\right)_{0} \sigma(x) d x u(y) d S_{y}\right| \\
= & \left|\int_{\Sigma}\left(S_{\phi, \delta, j} f_{j}\right)_{0}(y) u(y) d S_{y}\right| \\
& \leq\|u\|_{L_{2}(\Sigma)}\left\|S_{\phi, \delta, j} f_{j}\right\|_{L_{2}(\Sigma)} \\
& \leq C\|\phi\|_{K\left(C_{\mu_{+}}^{o}\right)}\|u\|_{L_{2}(\Sigma)}\left\|f_{j}\right\|_{\mathscr{P}_{+}} \quad \text { (by Lemma 5.3) } \\
& \leq C\|\phi\|_{K\left(C_{\mu_{+}}^{o}\right)}\|u\|_{L_{2}(\Sigma)} .
\end{aligned}
$$

Thus we get

$$
\left\|W_{\phi_{\delta}} u\right\|_{L_{2}(\Sigma)} \leq C\|\phi\|_{K\left(C_{\mu+}^{o}\right)}\|u\|_{L_{2}(\Sigma)}
$$

for all $u \in L_{2}(\Sigma)$ with compact support. Therefore the operators $W_{\phi_{\delta}}$ are Calderón-Zygmund operators, so

$$
\left\|W_{\phi_{\delta}} u\right\|_{L_{p}(\Sigma)} \leq C_{p}\|\phi\|_{K\left(C_{\mu+}^{o}\right)}\|u\|_{L_{p}(\Sigma)}
$$

for $u \in L_{p}(\Sigma)$.

We now prove the same estimates for $T_{\phi_{\delta}} u, u \in L_{p}(\Sigma, \mathscr{X})$, where

$$
\left(T_{\phi_{\delta}} u\right)(x)=\int_{\Sigma} \phi(x+\delta-y) n(y) u(y) d S_{y} .
$$

(We could not do this directly because $T_{\phi_{\delta}} u$ is not monogenic on $\Omega_{+}$.) 
Let $\left(v_{k}\right)_{k=1, \ldots, M}$ be a basis in $\mathscr{X}$. Then $n u=\sum_{k=1}^{M}(n u)_{k} v_{k}$ where $(n u)_{k}$ are scalar valued and $\sum_{k=1}^{M}\left\|(n u)_{k}\right\|_{p} \leq c\|n u\|_{p} \leq c\|u\|_{p}$. So

$$
\begin{aligned}
\left\|T_{\phi_{\delta}} u\right\|_{L_{p}(\Sigma)} & \leq \sum_{k=1}^{M}\left\|W_{\phi_{\delta}}(n u)_{k}\right\|_{L_{p}(\Sigma)} \leq C\|\phi\|_{K\left(C_{\mu+}^{o}\right)} \sum_{k=1}^{M}\left\|(n u)_{k}\right\|_{L_{p}(\Sigma)} \\
& \leq C\|\phi\|_{K\left(C_{\mu+}^{o}\right)}\|u\|_{L_{p}(\Sigma)} .
\end{aligned}
$$

We next prove that for $u \in L_{p}(\Sigma), T_{\phi_{\delta}} u$ converges in $L_{p}(\Sigma)$ as $\delta \rightarrow 0$.

Lemma 5.4. Let

$$
\Phi_{t}(x)=\frac{1}{\sigma_{n}}\left(\frac{\overline{x+t}}{|x+t|^{n+1}}-\frac{\overline{x-t}}{|x-t|^{n+1}}\right), \quad t>0, x \in S_{\pi / 2}^{o} .
$$

Then for $u \in L_{p}(\Sigma), \Phi_{t} * u \rightarrow u$ in $L_{p}(\Sigma)$ as $t \rightarrow 0$.

This is proved by noticing that

$$
\left|\Phi_{t}(x)\right| \leq C \frac{t}{\left(|\mathbf{x}|^{2}+t^{2}\right)^{(n+1) / 2}} \quad \text { and } \quad \int_{\mathbb{R}^{n}} \Phi_{t}(x) d x=1 .
$$

According to this lemma, we only have to prove $T_{\phi_{\delta}}\left(\Phi_{t} * u\right)$ converges in $L_{p}(\Sigma)$ as $\delta \rightarrow 0$ for fixed $t>0$ and $u$ compactly supported, Lipschitz continuous (for the set of such functions $\Phi_{t} * u$ is dense in $L_{p}(\Sigma)$, and the operators $T_{\phi_{\delta}}$ are uniformly bounded in $\delta$ ).

Notice that $T_{\phi_{\delta}}\left(\Phi_{t} * u\right)=\phi_{\delta+t} * u$ for $\delta<t$ and

$$
\left|\phi_{\delta+t}(x)-\phi_{\delta^{\prime}+t}(x)\right| \leq C \frac{\left|\delta-\delta^{\prime}\right|}{\left(|\mathbf{x}|^{2}+t^{2}\right)^{(n+1) / 2}} \quad \text { for } x \in S_{\mu}^{o} .
$$

We get $\left\|\phi_{\delta+t} * u-\phi_{\delta^{\prime}+t} * u\right\|_{L_{p}(\Sigma)} \rightarrow 0$ as $\delta, \delta^{\prime} \rightarrow 0$ which means $T_{\phi_{\delta}}\left(\Phi_{t} * u\right)$ converges in $L_{p}(\Sigma)$ as $\delta \rightarrow 0$. Hence there exists a bounded operator $T_{\phi}$ on $L_{p}(\Sigma)$ given by

$$
T_{\phi} u=\lim _{\delta \rightarrow 0} T_{\phi_{\delta}} u \text { for } u \in L_{p}(\Sigma)
$$

which satisfies the required estimate

$$
\left\|T_{\phi} u\right\|_{L_{p}(\Sigma)} \leq C\|\phi\|_{K\left(C_{\mu+}^{o}\right)}\|u\|_{L_{p}(\Sigma)} .
$$

Actually $T_{\phi} u=\sum_{k=1}^{M}\left(T_{\phi, k} u\right) v_{k}$ where

$$
\left(T_{\phi, k} u\right)(x)=\lim _{\delta \rightarrow 0} \int_{\Sigma} \phi(x-y+\delta)(n u)_{k}(y) d S_{y}=T_{\phi}\left(n^{-1}(n u)_{k}\right)(x)
$$

are right-monogenic functions, so we also get

$$
\begin{aligned}
\left\|N\left(T_{\phi} u\right)\right\|_{L_{p}(\Sigma)} & \leq C \sum_{k=1}^{M}\left\|N\left(T_{\phi, k} u\right)\right\|_{L_{p}(\Sigma)} \leq C \sum_{k=1}^{M}\left\|T_{\phi}\left(n^{-1}(n u)_{k}\right)\right\|_{L_{p}(\Sigma)} \\
& \leq C \sum_{k=1}^{M}\left\|n^{-1}(n u)_{k}\right\|_{L_{p}(\Sigma)} \leq C\|u\|_{L_{p}(\Sigma)}
\end{aligned}
$$


where $N$ is the nontangential maximal function. So the convergence is also almost everywhere. That is,

$$
T_{\phi} u(x)=\lim _{\delta \rightarrow 0} T_{\phi_{\delta}} u(x)
$$

for almost all $x \in \Sigma$, which is the first formula for $T_{\phi}$ given in Theorem 5.1.

To derive the second formula, we first consider functions $u$ which are compactly supported and Lipschitz continuous. Then for any $\varepsilon>0$

$$
\begin{aligned}
& T_{\phi_{\delta}} u(x)=\int_{\Sigma} \phi(x-y+\delta) n(y) u(y) d S_{y} \\
& \quad=\iint_{\substack{x-y \mid \geq \varepsilon \\
y \in \Sigma}} \phi(x-y+\delta) n(y) u(y) d S_{y}+\int_{\substack{|x-y| \leq \varepsilon \\
y \in \Sigma}} \phi(x-y+\delta) n(y) u(y) d S_{y} .
\end{aligned}
$$

Since

$$
\begin{aligned}
& \left|\int_{\substack{|x-y| \leq \varepsilon \\
y \in \Sigma}} \phi(x-y+\delta) n(y) u(y) d S_{y}-\int_{\substack{|x-y| \leq \varepsilon \\
y \in \Sigma}} \phi(x-y+\delta) n(y) d S_{y} u(x)\right| \\
& \quad=\left|\int_{\substack{|x-y| \leq \varepsilon \\
y \in \Sigma}} \phi(x-y+\delta) n(y)(u(y)-u(x)) d S_{y}\right| \\
& \quad \leq C \int_{\substack{|x-y| \leq \varepsilon \\
y \in \Sigma}} \frac{1}{|x-y+\delta|^{n}}|u(y)-u(x)| d S_{y} \\
& \quad \leq C\left\|u^{\prime}\right\|_{\infty} \iint_{\substack{|x-y| \leq \varepsilon \\
y \in \Sigma}} \frac{d S_{y}}{|x-y|^{n-1}} \leq C \varepsilon\left\|u^{\prime}\right\|_{\infty},
\end{aligned}
$$

(2) $\int \underset{\substack{|x-y| \leq \varepsilon \\ y \in \Sigma}}{ } \phi(x-y+\delta) n(y) d S_{y}=\int_{\substack{|x-y|=\varepsilon \\ y \in \Omega_{+}}} \phi(x-y+\delta) n(y) d S_{y}$, (by the right-monogenicity of $\phi$ ) and

(3) $\lim _{\varepsilon \rightarrow 0}\left\{\int_{\substack{|x-y|=\varepsilon \\ y \in \Omega_{+}}} \phi(x-y) n(y) d S_{y}-\underline{\phi}_{+}(\varepsilon n(x))\right\}=0 \quad$ a.e.

we get

$$
T_{\phi} u(x)=\lim _{\varepsilon \rightarrow 0}\left\{\iint_{\substack{|x-y|>\varepsilon \\ y \in \Sigma}} \phi(x-y) n(y) u(y) d S_{y}+\underline{\phi}_{+}(\varepsilon n(x)) u(x)\right\} \quad \text { a.e. }
$$

This gives that $T_{\phi}$ is a Calderón-Zygmund operator and so we can apply Theorem 5 in Chapter VII of [16] to conclude that, for all $u \in L_{p}(\Sigma)$, we have

$$
T_{\phi} u(x)=\lim _{\varepsilon \rightarrow 0}\left\{\iint_{\substack{|x-y|>\varepsilon \\ y \in \Sigma}} \phi(x-y) n(y) u(y) d S_{y}+\underline{\phi}_{+}(\varepsilon n(x)) u(x)\right\} \quad \text { a.e. }
$$

A similar proof applies to $\phi \in K\left(C_{\mu-}^{o}\right)$. This completes the proof of Theorem 5.1 .

Let us turn our attention to convolutions. Define $M\left(C_{\mu \pm}^{o}\right)=\left\{\phi \in K\left(C_{\mu \pm}^{o}\right)\right.$ : $\phi$ is left-monogenic as well $\}$. 
Theorem 5.5. Let $\phi \in K\left(C_{\mu+}^{o}\right)$ and $\psi \in M\left(C_{\mu+}^{o}\right)$.

(1) For $x \in C_{\mu+}^{o}$ define

$$
\begin{aligned}
(\phi * \psi)(x) & =\int_{\partial C_{\mu+}} \phi(x-y-\delta) n(y) \psi(y+\delta) d S_{y} \\
\quad \text { where } 0<\delta<\operatorname{dist}\left(x, \partial C_{\mu+}\right) & \\
& =\lim _{\varepsilon \rightarrow 0}\left\{\int_{\substack{H^{+}(\varepsilon, \mu) \\
|y|>\varepsilon}} \phi(x-y) n(y) \psi(y) d S_{y}+\underline{\phi}_{+}(\varepsilon) \psi(x)\right\}
\end{aligned}
$$

where $H^{+}(\varepsilon, \mu)=\left\{x+\varepsilon \tan \mu: x=x_{0}+\mathbf{x} \in \mathbb{R}^{n+1}, x_{0}=-|\mathbf{x}| \tan \mu\right\}$.

Then $\phi * \psi \in K\left(C_{\nu+}^{o}\right)$ for all $\nu<\mu$ and

$$
\|\phi * \psi\|_{K\left(C_{\nu+}^{o}\right)} \leq C\|\phi\|_{K\left(C_{\mu+}^{o}\right.}\|\psi\|_{K\left(C_{\mu+}^{o}\right)} .
$$

(2) $T_{\phi} \cdot T_{\psi}=T_{\phi * \psi}$.

(3) If $\psi_{1} \in M\left(C_{\mu+}^{o}\right)$, then $\psi * \psi_{1} \in M\left(C_{\nu+}^{o}\right)$ for all $\nu<\mu$, and $\phi *\left(\psi * \psi_{1}\right)=$ $(\phi * \psi) * \psi_{1}$.

Proof. (1) and (3) are easy to check.

To prove (2), we have

$$
\begin{gathered}
T_{\phi} \cdot T_{\psi} u(x)=\lim _{\delta \rightarrow 0+} \int_{\Sigma} \phi(x+\delta-y) n(y) \lim _{\varepsilon \rightarrow 0+} \int_{\Sigma} \psi(y+\varepsilon-z) n(z) u(z) d S_{z} d S_{y} \\
=\lim _{\delta \rightarrow 0+} \lim _{\varepsilon \rightarrow 0+} \int_{\Sigma}\left(\int_{\Sigma} \phi(x+\delta-y) n(y) \psi(y+\varepsilon-z) d S_{y}\right) n(z) u(z) d S_{z} .
\end{gathered}
$$

But

$$
\begin{aligned}
& \int_{\Sigma} \phi(x+\delta-y) n(y) \psi(y+\varepsilon-z) d S_{y}=\int_{\Sigma-z} \phi(x-z+\delta-y) n(y) \psi(y+\varepsilon) d S_{y} \\
& \quad=\int_{\partial C_{\mu+}} \phi(x-z+\delta-y) n(y) \psi(y+\varepsilon) d S_{y} \quad \text { (by using Cauchy's theorem) } \\
& \quad=(\phi * \psi)(x-z+\delta+\varepsilon) .
\end{aligned}
$$

Therefore

$$
T_{\phi} \cdot T_{\psi} u(x)=\lim _{\delta \rightarrow 0+} \int_{\Sigma}(\phi * \psi)(x+\delta-z) n(z) u(z) d S_{z}=T_{\phi * \psi} u(x) .
$$

Similarly we define $\phi * \psi$ for $\phi, \psi \in M\left(C_{\mu-}^{o}\right)$ and the corresponding results are true.

This theorem implies the following well-known results about the Cauchy operators $P_{ \pm}$and $C_{\Sigma}$ defined in Corollary 5.2.

Corollary 5.6. Let $\phi_{ \pm} \in M\left(C_{\mu \pm}^{o}\right)$. Then

$$
\begin{aligned}
& P_{+} T_{\phi_{+}}=T_{\phi_{+}} P_{+}=T_{\phi_{+}}, \quad P_{-} T_{\phi_{+}}=T_{\phi_{+}} P_{-}=0 \text {, } \\
& P_{-} T_{\phi_{-}}=T_{\phi_{-}} P_{-}=T_{\phi_{-}}, \quad P_{+} T_{\phi_{-}}=T_{\phi_{-}} P_{+}=0 \text {. }
\end{aligned}
$$




$$
\begin{gathered}
P_{+}{ }^{2}=P_{+}, \quad P_{-}{ }^{2}=P_{-}, \quad P_{+} P_{-}=P_{-} P_{+}=0, \quad C_{\Sigma}{ }^{2}=I . \\
T_{\phi+} T_{\phi-}=T_{\phi-} T_{\phi+}=0 .
\end{gathered}
$$

Proof. This follows immediately from Theorem 5.5 and the identities $k_{+} * \phi_{+}=$ $\phi_{+} * k_{+}=\phi_{+}$and $k_{-} * \phi_{-}=\phi_{-} * k_{-}=-\phi_{-}$.

\section{HARDY DECOMPOSITION OF $K\left(S_{\mu}^{o}\right)$}

Our proofs of Theorem 3.1 and Theorem 3.2 depend on the following decomposition results.

\section{Theorem 6.1.}

(1) Given $\phi_{ \pm} \in K\left(C_{\mu \pm}^{o}\right)$, let $\phi=\phi_{+}+\phi_{-}, \underline{\phi}=\underline{\phi}_{+}+\underline{\phi}_{-}$where $\underline{\phi}_{ \pm}$were defined in $\S 5$. Then $(\phi, \underline{\phi}) \in K\left(S_{\mu}^{o}\right)$ and

$$
\|(\phi, \underline{\phi})\|_{K\left(S_{\mu}^{o}\right)} \leq\left(\left\|\phi_{+}\right\|_{K\left(C_{\mu+}^{o}\right)}+\left\|\phi_{-}\right\|_{K\left(C_{\mu-}^{o}\right)}\right) .
$$

(2) Conversely, given $(\phi, \underline{\phi}) \in K\left(S_{\mu}^{o}\right)$ there exist unique functions $\phi_{+} \in$ $K\left(C_{\mu+}^{o}\right)$ and $\phi_{-} \in K\left(\bar{C}_{\mu-}^{o}\right)$ such that $\phi=\phi_{+}+\phi_{-}, \quad \underline{\phi}=\underline{\phi}_{+}+\underline{\phi}_{-}$. Moreover

$$
\left\|\phi_{ \pm}\right\|_{K\left(C_{\mu \pm}^{o}\right)} \leq c\|(\phi, \underline{\phi})\|_{K\left(S_{\mu}^{o}\right)}
$$

where $c$ depends on the dimension $n$ and $\mu$. Indeed, for $x \in C_{\mu \pm}^{o}$

$$
\phi_{ \pm}(x)= \pm \lim _{\varepsilon \rightarrow 0+} \frac{1}{\sigma_{n}}\left\{\int_{\substack{H^{ \pm}(\varepsilon, \mu) \\|y|>\varepsilon}} \phi(y) n(y) \frac{\overline{x-y}}{|x-y|^{n+1}} d S_{y}+\underline{\phi}(\varepsilon) \frac{\bar{x}}{|x|^{n+1}}\right\}
$$

where $H^{ \pm}(\varepsilon, \mu)$ are the $n$-surfaces defined by

$$
H^{ \pm}(\varepsilon, \mu)=\left\{x \pm \varepsilon \tan \mu: x=x_{0}+\mathbf{x} \in \mathbb{R}^{n+1}, x_{0}=\mp|\mathbf{x}| \tan \mu\right\}
$$

and $n(y)$ is the normal to the surface at $y$ which is directed upwards.

(3) $(\phi, \underline{\phi}) \in M\left(S_{\mu}^{o}\right)$ if and only if $\phi_{ \pm} \in M\left(C_{\mu \pm}^{o}\right)$.

Proof of Theorem 3.1. Define $T_{(\phi, \underline{ })}=T_{\phi_{+}}+T_{\phi_{-}}$by using the decomposition of Theorem $6.1(2)$. Then $T_{(\phi, \underline{)})}$ is a bounded linear operator on $L_{p}(\Sigma)$ which satisfies the formula and the bound given in Theorem 3.1.

Proof of Theorem 6.1.

(1) To see that

$$
\underline{\phi}_{ \pm}(R)-\underline{\phi}_{ \pm}(r)=\int_{r<|x|<R} \phi_{ \pm}(x) d S_{x},
$$

apply Cauchy's theorem to the monogenic functions $\phi_{ \pm}$. The bounds are straightforward. 
(2) To prove uniqueness, suppose that $\phi_{+}+\phi_{-}=0, \underline{\phi}_{+}+\underline{\phi}_{-}=0$. Then $\phi_{+}=-\phi_{-}$can be extended monogenically to $\mathbb{R}^{n+1} \backslash\{0\}$. Thus by Theorem 2.2,

$$
\phi_{+}(x)=c \frac{\bar{x}}{|x|^{n+1}}, \quad \phi_{-}=-c \frac{\bar{x}}{|x|^{n+1}}
$$

for some $c \in \mathbb{C}_{(n)}$. From the definition of $\underline{\phi}_{ \pm}$we obtain $\underline{\phi}_{+}=\underline{\phi}_{-}=\frac{c}{2}$, so $c=0$, and therefore $\phi_{+}=\phi_{-}=0$.

Let

$$
\phi_{ \pm}^{\varepsilon}(x)= \pm \frac{1}{\sigma_{n}}\left\{\int_{\substack{H^{ \pm}(\varepsilon, \mu) \\|y|>\varepsilon}} \phi(y) n(y) \frac{\overline{x-y}}{|x-y|^{n+1}} d S_{y}+\underline{\phi}(\varepsilon) \frac{\bar{x}}{|x|^{n+1}}\right\} .
$$

We prove that $\lim \phi_{ \pm}^{\varepsilon}(x)$ exist when $\varepsilon \rightarrow 0$, then define $\phi_{ \pm}(x)=\lim _{\varepsilon \rightarrow 0} \phi_{ \pm}^{\varepsilon}(x)$. We only look at $\phi_{+}^{\varepsilon}(x)$. For fixed

$$
\begin{aligned}
& x \in C_{\mu+}^{o} \text { and } 0<\varepsilon_{2}<\varepsilon_{1}<\min \left(\frac{1}{2}|x|, \operatorname{dist}\left(x, \partial C_{\mu+}^{o}\right)\right), \\
& \phi_{+}^{\varepsilon_{1}}(x)-\phi_{+}^{\varepsilon_{2}}(x)=\frac{1}{\sigma_{n}}\left(\int_{H^{ \pm}\left(\varepsilon_{1}, \mu\right)}-\int_{\substack{|y|>\varepsilon_{1} \\
H^{ \pm}\left(\varepsilon_{2}, \mu\right) \\
|y|>\varepsilon_{2}}}\right) \phi(y) n(y) \frac{\overline{x-y}}{|x-y|^{n+1}} d S_{y} \\
& +\left(\underline{\phi}\left(\varepsilon_{1}\right)-\underline{\phi}\left(\varepsilon_{2}\right)\right) \frac{\bar{x}}{|x|^{n+1}} \\
& =-\frac{1}{\sigma_{n}} \int_{\substack{\varepsilon_{2}<|y|<\varepsilon_{1} \\
y \in \mathbb{R}^{n}}} \phi(y)\left(\frac{\overline{x-y}}{|x-y|^{n+1}}-\frac{\bar{x}}{|x|^{n+1}}\right) d y .
\end{aligned}
$$

Hence

$$
\begin{aligned}
\left|\phi_{+}^{\varepsilon_{1}}(x)-\phi_{+}^{\varepsilon_{2}}(x)\right| & \leq \frac{C}{\sigma_{n}} \int_{\varepsilon_{2}<|y|<\varepsilon_{1}} \frac{1}{|y|^{n}}\left|\frac{\overline{x-y}}{|x-y|^{n+1}}-\frac{\bar{x}}{|x|^{n+1}}\right| d y \\
& \leq \frac{C}{\sigma_{n}} \int_{\varepsilon_{2}<|y|<\varepsilon_{1}} \frac{1}{|y|^{n}} \frac{1}{|x|^{n+1}}|y| d y \\
& =\frac{C}{\sigma_{n}} \frac{1}{|x|^{n+1}}\left(\varepsilon_{1}-\varepsilon_{2}\right) \rightarrow 0 \quad \text { as } \varepsilon_{1} \rightarrow 0, \varepsilon_{2} \rightarrow 0 .
\end{aligned}
$$

Thus $\left\{\phi_{+}^{\varepsilon}(x)\right\}_{\varepsilon}$ is a Cauchy net for $x \in C_{\mu+}^{o}$ and therefore $\phi_{+}(x)=$ $\lim _{\varepsilon \rightarrow 0} \phi_{+}^{\varepsilon}(x)$ exists pointwise on $C_{\mu+}^{o}$. Furthermore from the proof we can see that the convergence is uniform on any compact subsets of $C_{\mu+}^{o}$. So $\phi_{+}$is rightmonogenic because it can be uniformly approximated by the right-monogenic functions $\phi_{+}^{\varepsilon}$ on every compact subset of $C_{\mu+}^{o}$. Similarly $\phi_{-}$is right monogenic.

Let $E(\varepsilon, \mu)$ be the set of points $x$ between $H^{+}(\varepsilon, \mu)$ and $H^{-}(\varepsilon, \mu)$ with $|x|>\varepsilon$. From the Cauchy integral formula, $\phi_{+}^{\varepsilon}(x)+\phi_{-}^{\varepsilon}(x)=\phi(x)$. On letting $\varepsilon \rightarrow 0$ we get

$$
\phi_{+}(x)+\phi_{-}(x)=\phi(x), \quad x \in S_{\mu}^{o} .
$$


To prove $\underline{\phi}_{+}(r)+\underline{\phi}_{-}(r)=\underline{\phi}(r)$, we look at $\phi_{ \pm}^{\varepsilon}(r)$ when $\varepsilon<r$. By Cauchy's theorem, we have

$$
\begin{array}{ll}
\phi_{+}^{\varepsilon}(x)=\frac{1}{\sigma_{n}}\left\{\int_{\substack{y \in \mathbb{R}^{n} \\
|y|>\varepsilon}} \phi(y) \frac{\overline{x-y}}{|x-y|^{n+1}} d y+\underline{\phi}(\varepsilon) \frac{\bar{x}}{\left.|x|^{n+1}\right\},},\right. & x \in \mathbb{R}_{+}^{n+1}, \\
\phi_{-}^{\varepsilon}(x)=-\frac{1}{\sigma_{n}}\left\{\int_{\substack{y \in \mathbb{R}^{n} \\
|y|>\varepsilon}} \phi(y) \frac{\overline{x-y}}{|x-y|^{n+1}} d y+\underline{\phi}(\varepsilon) \frac{\bar{x}}{\left.|x|^{n+1}\right\},}\right. & x \in \mathbb{R}_{-}^{n+1} .
\end{array}
$$

Then

$$
\begin{aligned}
& \underline{\phi}_{+}^{\varepsilon}(r)=\int_{\substack{x_{0}>0 \\
|x|=r}}\left(\frac{1}{\sigma_{n}} \int_{\substack{y \in \mathbb{R}^{n} \\
|y|>\varepsilon}} \phi(y) \frac{\overline{x-y}}{|x-y|^{n+1}} d y\right) n(x) d S_{x}+\frac{1}{2} \underline{\phi}(\varepsilon), \\
& \underline{\phi}_{-}^{\varepsilon}(r)=\int_{\substack{|x|=r \\
x_{0}<0}}\left(\frac{1}{\sigma_{n}} \int_{\substack{y \in \mathbb{R}^{n} \\
|y|>\varepsilon}} \phi(y) \frac{\overline{x-y}}{|x-y|^{n+1}} d y\right) n(x) d S_{x}+\frac{1}{2} \underline{\phi}(\varepsilon),
\end{aligned}
$$

where $n(x)$ is the outward normal to the n-sphere $\left\{x \in \mathbb{R}^{n+1}:|x|=r\right\}$. Thus

$$
\begin{aligned}
\underline{\phi}_{+}^{\varepsilon}(r)+\underline{\phi}_{-}^{\varepsilon}(r) & =\int_{|x|=r}\left(\frac{1}{\sigma_{n}} \int_{\substack{y \in \mathbb{R}^{n} \\
|y|>\varepsilon}} \phi(y) \frac{\overline{x-y}}{|x-y|^{n+1}} d y\right) n(x) d S_{x}+\underline{\phi}(\varepsilon) \\
& =\int_{\substack{y \in \mathbb{R}^{n} \\
|y|>\varepsilon}} \phi(y)\left(\frac{1}{\sigma_{n}} \int_{|x|=r} \frac{\overline{x-y}}{|x-y|^{n+1}} n(x) d S_{x}\right) d y+\underline{\phi}(\varepsilon) .
\end{aligned}
$$

By Cauchy's integral formula,

$$
\frac{1}{\sigma_{n}} \int_{|x|=r} \frac{\overline{x-y}}{|x-y|^{n+1}} n(x) d S_{x}= \begin{cases}1, & |y|<r, \\ 0, & |y|>r .\end{cases}
$$

Hence

$$
\underline{\phi}_{+}^{\varepsilon}(r)+\underline{\phi}_{-}^{\varepsilon}(r)=\int_{\varepsilon<|y|<r} \phi(y) d y+\underline{\phi}(\varepsilon)=\underline{\phi}(r) .
$$

On letting $\varepsilon \rightarrow 0$ we get $\underline{\phi}_{+}(r)+\underline{\phi}_{-}(r)=\underline{\phi}(r)$.

To prove the estimates, for $x \in \mathbb{R}_{+}^{n+1},|x|>2 \varepsilon$, we have

$$
\begin{aligned}
& \int_{\substack{H^{+}(\varepsilon, \mu) \\
|y|>\varepsilon}} \phi(y) n(y) \frac{\overline{x-y}}{|x-y|^{n+1}} d S_{y} \\
& \quad=\int_{\substack{H^{+}(\varepsilon, \mu) \\
H^{+}|y|<\frac{1}{2}|x|}} \phi(y) n(y) \frac{\overline{x-y}}{|x-y|^{n+1}} d S_{y}+\int_{H_{\frac{1}{2}|x|<|y|}^{+}(\varepsilon)} \phi(y) n(y) \frac{\overline{x-y}}{|x-y|^{n+1}} d S_{y} \\
& \quad=I_{1}+I_{2} .
\end{aligned}
$$

For $I_{2}$, notice that when $y \in H^{+}(\varepsilon, \mu)$, and $\frac{1}{2}|x|<|y|$, then $|x-y|>|y| \sin \mu$.

Therefore

$$
\begin{aligned}
\left|I_{2}\right| & \leq \sigma_{n}^{-1}\|(\phi, \underline{\phi})\|_{K\left(S_{\mu}^{o}\right)} \int_{\substack{\left.H^{+} \mid \varepsilon, \mu\right) \\
2}|y|} \frac{1}{|x-y|^{n}} \frac{1}{|y|^{n}} d S_{y} \\
& \leq \sigma_{n}^{-1}\|(\phi, \underline{\phi})\|_{K\left(S_{\mu}^{o}\right)} \int_{\substack{\frac{1}{2}|x|<|y| \\
H^{+}(\varepsilon, \mu)}} \frac{1}{(\sin \mu)^{n}} \frac{1}{|y|^{2 n}} d S_{y} \\
& \leq c\|(\phi, \underline{\phi})\|_{K\left(S_{\mu}^{o}\right)} /|x|^{n} .
\end{aligned}
$$


For $I_{1}$, we want to prove that

$$
\left|I_{1}+\underline{\phi}(\varepsilon) \frac{\bar{x}}{|x|^{n+1}}\right| \leq c\|(\phi, \underline{\phi})\|_{K\left(S_{\mu}^{o}\right)} /|x|^{n} .
$$

Since

$$
\begin{aligned}
& \underline{\phi}(\varepsilon)=\underline{\phi}\left(\frac{1}{2}|x|\right)-\int_{\substack{\varepsilon<|y|<\frac{1}{2}|x| \\
y \in \mathbb{R}^{n}}} \phi(y) d y, \\
& I_{1}=\int_{\substack{\varepsilon<|y|<\frac{1}{2}|x| \\
y \in \mathbb{R}^{n}}} \phi(y) \frac{\overline{x-y}}{|x-y|^{n+1}} d y+\int_{S_{\mu}\left(\frac{1}{2}|x|\right)} \phi(y) n(y) \frac{\overline{x-y}}{|x-y|^{n+1}} d S_{y}
\end{aligned}
$$

where $S_{\mu}(r)=\left\{x=x_{0}+\mathbf{x} \in \mathbb{R}^{n+1},|x|=r, 0<-x_{0} /|\mathbf{x}|<\tan \mu\right\}$ and

$$
\begin{aligned}
& \left|\int_{S_{\mu}\left(\frac{1}{2}|x|\right)} \phi(y) n(y) \frac{\overline{x-y}}{|x-y|^{n+1}} d S_{y}\right| \\
& \quad \leq \sigma_{n}^{-1}\|(\phi, \underline{\phi})\|_{K\left(S_{\mu}^{o}\right)} \int_{S_{\mu}\left(\frac{1}{2}|x|\right)} \frac{1}{|x-y|^{n}} \frac{1}{|y|^{n}} d S_{y} \\
& \quad \leq c\|(\phi, \underline{\phi})\|_{K\left(S_{\mu}^{o}\right)} /|x|^{n} .
\end{aligned}
$$

Therefore

$$
\begin{aligned}
\mid I_{1}+ & \underline{\phi}(\varepsilon) \frac{\bar{x}}{|x|^{n+1}} \mid \\
\leq & \left|\int_{\substack{\varepsilon<|y|<\frac{1}{2}|x| \\
y \in \mathbb{R}^{n}}} \phi(y)\left(\frac{\overline{x-y}}{|x-y|^{n+1}}-\frac{\bar{x}}{|x|^{n+1}}\right) d y\right|+c\|(\phi, \underline{\phi})\|_{K\left(S_{\mu}^{o}\right)} /|x|^{n} \\
& +\left|\underline{\phi}\left(\frac{1}{2}|x|\right) \frac{\bar{x}}{|x|^{n+1}}\right| \\
& \leq c \sigma_{n}^{-1}\|(\phi, \underline{\phi})\|_{K\left(S_{\mu}^{o}\right)} \int_{\substack{y \in|y|<\frac{1}{2}|x| \\
|x|^{n+1}}} \frac{1}{|y|^{n-1}} d y+c\|(\phi, \underline{\phi})\|_{K\left(S_{\mu}^{o}\right)} /|x|^{n} \\
& \leq c\|(\phi, \underline{\phi})\|_{K\left(S_{\mu}^{o}\right)} /|x|^{n} .
\end{aligned}
$$

Thus we have proved that giving any $\varepsilon>0$, for $x \in \mathbb{R}_{+}^{n+1},|x|>2 \varepsilon$,

$$
\begin{aligned}
& \left|\int_{\substack{H^{+}(\varepsilon, \mu) \\
|y|>\varepsilon}} \phi(y) n(y) \frac{\overline{x-y}}{|x-y|^{n+1}} d S_{y}+\underline{\phi(\varepsilon)} \frac{\bar{x}}{|x|^{n+1}}\right| \\
& \leq c\|(\phi, \underline{\phi})\|_{K\left(S_{\mu}^{o}\right)} /|x|^{n}
\end{aligned}
$$

with $c$ a constant depending on $n$ and $\mu$. On letting $\varepsilon \rightarrow 0$, we get

$$
\left|\phi_{+}(x)\right| \leq \frac{c}{\sigma_{n}}\|(\phi, \underline{\phi})\|_{K\left(S_{\mu}^{o}\right)} /|x|^{n}, \quad x \in \mathbb{R}_{+}^{n+1} .
$$


Similarly we get

$$
\left|\phi_{-}(x)\right| \leq \frac{c}{\sigma_{n}}\|(\phi, \underline{\phi})\|_{K\left(S_{\mu}^{o}\right)} /|x|^{n}, \quad x \in \mathbb{R}_{-}^{n+1} .
$$

These estimates can easily be extended from the half-spaces $\mathbb{R}_{ \pm}^{n+1}$ to the cones $C_{\mu \pm}^{o}$ by using the identity $\phi_{+}+\phi_{-}=\phi$. For, when $x \in C_{\mu+}^{o} \backslash \mathbb{R}_{+}^{n+1}$, then

$$
\left|\phi_{+}(x)\right| \leq\left|\phi_{-}(x)\right|+|\phi(x)| \leq \frac{c}{\sigma_{n}}\|(\phi, \underline{\phi})\|_{K\left(S_{\mu}^{o}\right)} /|x|^{n}
$$

and when $x \in C_{\mu-}^{o} \backslash \mathbb{R}_{-}^{n+1}$, then

$$
\left|\phi_{-}(x)\right| \leq\left|\phi_{+}(x)\right|+|\phi(x)| \leq \frac{c}{\sigma_{n}}\|(\phi, \underline{\phi})\|_{K\left(S_{\mu}^{o}\right)} /|x|^{n}
$$

Therefore

$$
\left\|\phi_{ \pm}\right\|_{K\left(C_{\mu \pm}^{o}\right)} \leq c\|(\phi, \underline{\phi})\|_{K\left(S_{\mu}^{o}\right)}
$$

This completes the proof of (2).

(3) Let $(\phi, \underline{\phi}) \in M\left(S_{\mu}^{o}\right)$. We have to prove that $\phi_{ \pm}$are left-monogenic as well as right-monogenic. Just as we defined the right-monogenic functions $\phi_{ \pm}$ on $C_{\mu \pm}^{o}$, we can also define left-monogenic functions ${ }^{L} \phi_{ \pm}(x)=\lim _{\varepsilon \rightarrow 0}{ }^{L} \phi_{ \pm}^{\varepsilon}(x)$ by

$$
{ }^{L} \phi_{ \pm}^{\varepsilon}(x)= \pm \frac{1}{\sigma_{n}}\left\{\int_{\substack{H^{ \pm}(\varepsilon, \mu) \\|y|>\varepsilon}} \frac{\overline{x-y}}{|x-y|^{n+1}} n(y) \phi(y) d S_{y}+\frac{\bar{x}}{|x|^{n+1}} \underline{\phi}(\varepsilon)\right\}
$$

for $x \in C_{\mu \pm}^{o}$. So what we need to prove is that ${ }^{L} \phi_{ \pm}(x)=\phi_{ \pm}(x)$. Let us consider $\phi_{+}$. If $x \in \mathbb{R}_{+}^{n+1}$ and $\varepsilon<\frac{1}{2}|x|$, then

$$
\begin{array}{r}
\phi_{+}^{\varepsilon}(x)-{ }^{L} \phi_{+}^{\varepsilon}(x)=\frac{1}{\sigma_{n}} \int_{\substack{y \in \mathbb{R}^{n} \\
|y|>\varepsilon}}(\phi(y) \\
\left.|x-y|^{n+1}-\frac{\overline{x-y}}{|x-y|^{n+1}} \phi(y)\right) d y \\
+\frac{1}{\sigma_{n}}\left(\underline{\phi}(\varepsilon) \frac{\bar{x}}{|x|^{n+1}}-\frac{\bar{x}}{|x|^{n+1}} \phi(\varepsilon)\right) .
\end{array}
$$

It follows from condition $(*)$ in the definition of $M\left(S_{\mu}^{o}\right)$ that

$$
\underline{\phi}(\varepsilon) \bar{x}-\bar{x} \underline{\phi}(\varepsilon)=-\int_{\substack{|y|=\varepsilon \\ y \in \mathbb{R}^{n}}}\langle y, x\rangle(\phi(y) n(y)-n(y) \phi(y)) d y .
$$


Also

$$
\begin{aligned}
& \int_{\substack{y \in \mathbb{R}^{n} \\
|y|>\varepsilon}}\left(\phi(y) \frac{\overline{x-y}}{|x-y|^{n+1}}-\frac{\overline{x-y}}{|x-y|^{n+1}} \phi(y)\right) d y \frac{-1}{n-1} \int_{\substack{y \in \mathbb{R}^{n} \\
|y|>\varepsilon}}\left(\phi(y) D_{y}\left(\frac{1}{|x-y|^{n-1}}-\frac{1}{|x|^{n-1}}\right)\right. \\
&\left.\quad-D_{y}\left(\frac{1}{|x-y|^{n-1}}-\frac{1}{|x|^{n-1}}\right) \phi(y)\right) d y \\
&=\frac{-1}{n-1} \int_{\substack{y \in \mathbb{R}^{n} \\
|y|>\varepsilon}}\left\{\left(\phi(y)\left(\frac{1}{|x-y|^{n-1}}-\frac{1}{|x|^{n-1}}\right)\right) \mathbf{D}_{y}\right. \\
&\left.\quad-\mathbf{D}_{y}\left(\phi(y)\left(\frac{1}{|x-y|^{n-1}}-\frac{1}{|x|^{n-1}}\right)\right)\right\} d y \\
&=\frac{-1}{n-1} \int_{\substack{y \in \mathbb{R}^{n} \\
|y|=\varepsilon}}(\phi(y) n(y)-n(y) \phi(y))\left(\frac{1}{|x-y|^{n-1}}-\frac{1}{|x|^{n-1}}\right) d y .
\end{aligned}
$$

Thus

$$
\begin{aligned}
\phi_{+}^{\varepsilon}(x)-{ }^{L} \phi_{+}^{\varepsilon}(x)=\frac{-1}{(n-1) \sigma_{n}} \int_{\substack{y \in \mathbb{R}^{n} \\
|y|=\varepsilon}}(\phi(y) n(y)-n(y) \phi(y)) \\
\quad \times\left(\frac{1}{|x-y|^{n-1}}-\frac{1}{|x|^{n-1}}+(n-1) \frac{\langle y, x\rangle}{|x|^{n+1}}\right) d y .
\end{aligned}
$$

But for $|y|=\varepsilon<\frac{1}{2}|x|$,

$$
\left|\frac{1}{|x-y|^{n-1}}-\frac{1}{|x|^{n-1}}+(n-1) \frac{\langle y, x\rangle}{|x|^{n+1}}\right| \leq \frac{C}{|x|^{n+1}} \varepsilon^{2}
$$

and

$$
|\phi(y) n(y)-n(y) \phi(y)| \leq C \varepsilon^{-n}
$$

Hence

$$
\begin{aligned}
& \left|\phi_{+}^{\varepsilon}(x)-{ }^{L} \phi_{+}^{\varepsilon}(x)\right| \leq \frac{1}{(n-1) \sigma_{n}} \int_{\substack{y \in \mathbb{R}^{n} \\
|y|=\varepsilon}} \varepsilon^{-n+2}|x|^{-(n+1)} d y \\
& \leq C|x|^{-(n+1)} \varepsilon \rightarrow 0 \text { as } \varepsilon \rightarrow 0 \text {. }
\end{aligned}
$$

Therefore $\phi_{+}={ }^{L} \phi_{+}$, so we conclude that $\phi_{+}$is left-monogenic on $\mathbb{R}_{+}^{n+1}$ and hence on $C_{\mu+}^{o+}$.

In order to prove the converse we need the following lemma.

Lemma 6.2. Given $(\phi, \underline{\phi}) \in K\left(S_{\mu}^{o}\right)$, suppose $\phi$ is left-as well as right-monogenic.

Let

$$
F(r, x)=\int_{\substack{|y|=r \\ y \in \mathbb{R}^{n}}}\langle y, x\rangle(\phi(y) n(y)-n(y) \phi(y)) d y-(\underline{\phi}(r) x-x \underline{\phi}(r)), \quad x \in \mathbb{R}^{n}
$$


Then $F(r, x)$ is actually independent of $r>0$.

Proof. For $0<r<R$,

$$
\begin{aligned}
F(R, & x)-F(r, x) \\
= & \int_{\substack{|y|=R \\
y \in \mathbb{R}^{n}}}\langle y, x\rangle(\phi(y) n(y)-n(y) \phi(y)) d y \\
& -\int_{\substack{|y|=r \\
y \in \mathbb{R}^{n}}}\langle y, x\rangle(\phi(y) n(y)-n(y) \phi(y)) d y-(\underline{\phi}(R)-\underline{\phi}(r)) x \\
& +x(\underline{\phi}(R)-\underline{\phi}(r)) \\
= & \int_{\substack{r<|y|<R \\
y \in \mathbb{R}^{n}}}\left\{(\langle y, x\rangle \phi(y)) \mathbf{D}_{y}-\mathbf{D}_{y}(\langle y, x\rangle \phi(y))\right\} d y \\
& -(\underline{\phi}(R)-\underline{\phi}(r)) x+x(\underline{\phi}(R)-\underline{\phi}(r)) \\
= & \int_{\substack{r<|y|<R \\
y \in \mathbb{R}^{n}}}(\phi(y) x-x \phi(y)) d y-(\underline{\phi}(R)-\underline{\phi}(r)) x+x(\underline{\phi}(R)-\underline{\phi}(r))=0
\end{aligned}
$$

(as $\left.\mathbf{D}_{y} \phi=-\partial \phi / \partial y_{0}=\phi \mathbf{D}_{y}\right)$. So $F(r, x)$ is independent of $r$.

What remains is to show that if $\phi_{ \pm} \in M\left(C_{\mu \pm}^{o}\right)$, then $(\phi, \underline{\phi}) \in M\left(S_{\mu}^{o}\right)$. Clearly $\phi$ is left-monogenic. Let us check that $\left(\phi_{+}, \underline{\phi}_{+}\right)$satisfies $(*)$. Since $\phi_{+}$equals its own left- and right-monogenic extensions, we see that $\left(\phi_{+}\right)_{+}^{\varepsilon}(x)-$ ${ }^{L}\left(\phi_{+}\right)_{+}^{\varepsilon}(x) \rightarrow 0$ for $x \in C_{\mu+}^{o}$. Especially for $x \in \mathbb{R}_{+}^{n+1}$,

$$
\begin{aligned}
\lim _{\varepsilon \rightarrow 0} \frac{1}{\sigma_{n}}\left\{\int _ { \substack { y \in \mathbb { R } ^ { n } \\
| y | > \varepsilon } } \left(\phi_{+}(y) \frac{\overline{x-y}}{|x-y|^{n+1}}\right.\right. & \left.-\frac{\overline{x-y}}{|x-y|^{n+1}} \phi_{+}(y)\right) d S_{y} \\
& \left.+\underline{\phi}_{+}(\varepsilon) \frac{\bar{x}}{|x|^{n+1}}-\frac{\bar{x}}{|x|^{n+1}} \underline{\phi}_{+}(\varepsilon)\right\}=0
\end{aligned}
$$

Notice that by Gauss's integral formula and the identity $\mathbf{D}_{y} \phi_{+}=\phi_{+} \mathbf{D}_{y}$, it follows as before that

$$
\begin{aligned}
& \frac{1}{\sigma_{n}} \int_{\substack{y \in \mathbb{R}^{n} \\
|y|>\varepsilon}}\left(\phi_{+}(y) \frac{\overline{x-y}}{|x-y|^{n+1}}-\frac{\overline{x-y}}{|x-y|^{n+1}} \phi_{+}(y)\right) d S_{y} \\
& \quad=\frac{1}{(n-1) \sigma_{n}} \int_{\substack{|y|=\varepsilon \\
y \in \mathbb{R}^{n}}}\left(\phi_{+}(y) n(y)-n(y) \phi_{+}(y)\right) \frac{1}{|x-y|^{n-1}} d S_{y} .
\end{aligned}
$$

Since for fixed $x$,

$$
\frac{1}{|x-y|^{n-1}}=\frac{1}{|x|^{n-1}}-(n-1) \frac{\langle x, y\rangle}{|x|^{n}}+\frac{o(\varepsilon)}{|x|^{n+1}}
$$

we have

$$
\int_{\substack{|y|=\varepsilon \\ y \in \mathbb{R}^{n}}}\langle y, x\rangle\left(\phi_{+}(y) n(y)-n(y) \phi_{+}(y)\right) d S_{y}-\left(\underline{\phi}_{+}(\varepsilon) x-x \underline{\phi}_{+}(\varepsilon)\right) \rightarrow 0
$$

as $\varepsilon \rightarrow 0$. By Lemma 6.2 it equals zero and therefore $\left(\phi_{+}, \underline{\phi}_{+}\right)$satisfies $(*)$. Similarly $\left(\phi_{-}, \underline{\phi}_{-}\right)$satisfies $(*)$. 
This finishes the proof of Theorem 6.1.

We finish this section with a proof of Theorem 3.2. Given $(\phi, \phi),(\psi, \psi) \in$ $M\left(S_{\mu}^{o}\right)$, define $(\phi, \underline{\phi}) *(\psi, \underline{\psi})=\left(\phi_{+} * \psi_{+}+\phi_{-} * \psi_{-}, \underline{\left(\phi_{+} * \psi_{+}\right)}+\underline{\left(\phi_{-} * \psi_{-}\right)}\right)$.

Then from Theorem 5.4 and Theorem 6.1, $(\phi, \underline{\phi}) *(\psi, \underline{\psi}) \in M\left(S_{\nu}^{o}\right)$ for all $\nu<\mu$ and

$$
\|(\phi, \underline{\phi}) *(\psi, \underline{\psi})\|_{K\left(S_{\nu}^{o}\right)} \leq C\|(\phi, \underline{\phi})\|_{K\left(S_{\mu}^{o}\right)}\|(\psi, \underline{\psi})\|_{K\left(S_{\mu}^{o}\right)} .
$$

(In particular $M\left(S_{\omega}\right)$ is an algebra under the *-product.) Moreover,

$$
\begin{aligned}
T_{(\phi, \underline{\phi})} \cdot T_{(\psi \underline{\psi})} & =\left(T_{\phi_{+}}+T_{\phi_{-}}\right)\left(T_{\psi_{+}}+T_{\psi_{-}}\right) \\
& =T_{\phi_{+}} \cdot T_{\psi_{+}}+T_{\phi_{-}} \cdot T_{\psi_{-}} \quad(\text { from Corollary 5.6) } \\
& =T_{\phi_{+} * \psi_{+}}+T_{\phi_{-} * \psi_{-}} \quad(\text { from Theorem 5.5) } \\
& =T_{(\phi, \underline{\phi}) *(\psi, \underline{\psi})} .
\end{aligned}
$$

\section{Condition $(*)$}

Our aim in this final section is to show that condition $(*)$ is not very restrictive.

Theorem 7.1. Given $(\phi, \underline{\phi}) \in K\left(S_{\mu}^{o}\right)$ with $\phi$ right-monogenic as well, there exists a constant $v \in \mathbb{R}_{(n)}\left(\right.$ or $\left.\mathbb{C}_{(n)}\right)$ such that $(\phi, \underline{\phi}-v) \in M\left(S_{\mu}^{o}\right)$ and $|v| \leq$ $C\|(\phi, \underline{\phi})\|_{K\left(S_{\mu}^{o}\right)}$, where $C$ is a constant which depends only on $n$.

Consequently there exist $\phi_{ \pm} \in M\left(C_{\mu \pm}^{o}\right)$ such that $\left\|\phi_{ \pm}\right\|_{K\left(C_{\mu \pm}^{o}\right)} \leq C\|(\phi, \underline{\phi})\|_{K\left(S_{\mu}^{o}\right)}$ and $T_{(\phi, \phi)}=T_{\phi_{+}}+T_{\phi_{-}}-v I$.

To prove this, we need the following results about Clifford algebras.

Lemma 7.2. Let $u_{k} \in \mathbb{R}_{(n)}$ (or $\left.\mathbb{C}_{(n)}\right)$ for $1 \leq k \leq n$. Then there exists $v \in \mathbb{R}_{(n)}$ (or $\mathbb{C}_{(n)}$ ) such that $v e_{k}-e_{k} v=u_{k}$ for all $k$ with $1 \leq k \leq n$ if and only if

$$
e_{k} u_{j}+e_{j} u_{k}+u_{j} e_{k}+u_{k} e_{j}=0 \quad \text { whenever } 1 \leq j \leq n \text { and } 1 \leq k \leq n \text {. }
$$

One solution is given by

$$
v=\sum_{j=1}^{n} \sum_{N=0}^{n} \alpha_{N}{ }^{-1} e_{j} u_{j, N}
$$

where $u_{j, N}=\sum_{|S|=N} u_{j, S} e_{S}$, and $\alpha_{N}=2(n+1-N)$ if $N$ is even and $\alpha_{N}=$ $2(N+1)$ if $N$ is odd.

Proof. It is easy to check that (7.1) holds if $u_{k}=v e_{k}-e_{k} v$, whether or not $j=k$.

For the converse, suppose that the $u_{k}$ satisfies (7.1), in which case the $u_{k, N}$ do too. This is because $e_{k} u_{j, N}+u_{j, N} e_{k}$ is of degree $N+(-1)^{N}$, so there is no mixing of degrees involved in (7.1). 
Let $w_{N}=\sum_{j=1}^{n} e_{j} u_{j, N}$. Then, for $1 \leq k \leq n$,

$$
\begin{aligned}
w_{N} e_{k}-e_{k} w_{N} & =\sum_{j=1}^{n}\left(e_{j} u_{j, N} e_{k}-e_{k} e_{j} u_{j, N}\right) \\
& =\sum_{j=1}^{n} e_{j}\left(u_{j, N} e_{k}+e_{k} u_{j, N}\right)+2 u_{k, N} \\
& =-\sum_{j=1}^{n} e_{j}\left(e_{j} u_{k, N}+u_{k, N} e_{j}\right)+2 u_{k, N} \\
& =(n+2) u_{k, N}-\sum_{|S|=N} u_{k, S}\left\{\sum_{j \in S} e_{j} e_{S} e_{j}+\sum_{j \notin S} e_{j} e_{S} e_{j}\right\} \\
& =(n+2) u_{k, N}-\sum_{|S|=N} u_{k, S}\left\{(-1)^{N} N+(-1)^{N+1}(n-N)\right\} e_{S} \\
& =\alpha_{N} u_{k, N}
\end{aligned}
$$

and so $v=\sum_{N=0}^{n} \alpha_{N}{ }^{-1} w_{N}$ as required.

Lemma 7.3. Given a $C^{1}$-function $F$ on $\mathbb{R}^{n} \backslash\{0\}$ which satisfies $\mathbf{D} F-F \mathbf{D}=0$, define

$$
u_{k}=\int_{|y|=1} y_{k}(F(y) y-y F(y)) d S_{y}, \quad 1 \leq k \leq n .
$$

Then the $u_{k}$ satisfy (7.1).

Proof. First consider (7.1) with $j=k$. (For ease of notation, take $j=k=1$.) Then

$$
\begin{aligned}
e_{1} u_{1}+u_{1} e_{1} & =\int_{|y|=1} y_{1}\left\{e_{1}(F(y) y-y F(y))+(F(y) y-y F(y)) e_{1}\right\} d S_{y} \\
& =\int_{|y|=1} y_{1}\left\{e_{1}\left(F(y) y^{+}-y^{+} F(y)\right)+\left(F(y) y^{+}-y^{+} F(y)\right) e_{1}\right\} d S_{y} \\
\text { where } y^{+}=y_{2} e_{2}+y_{3} e_{3}+\cdots+y_{n} e_{n} & \\
= & \int_{\substack{|y| \leq 1 \\
y_{1} \neq 0}} y_{1}\left\{e_{1}\left(F \mathbf{D}^{+}(y)-\mathbf{D}^{+} F(y)\right)+\left(F \mathbf{D}^{+}(y)-\mathbf{D}^{+} F(y)\right) e_{1}\right\} d S_{y} \\
& \left(\text { where } \mathbf{D}^{+}=\sum_{k=2}^{n} \frac{\partial}{\partial y_{k}} e_{k}\right)
\end{aligned}
$$

(using Gauss's theorem on the $(n-1)$-balls $y_{1}=\alpha,\left|y^{+}\right| \leq \sqrt{1-\alpha^{2}}$ )

$$
\begin{aligned}
=-\int_{\substack{|y| \leq 1 \\
y_{1} \neq 0}} y_{1}\left\{e _ { 1 } \left(\frac{\partial F}{\partial x_{1}}(y) e_{1}-\right.\right. & \left.e_{1} \frac{\partial F}{\partial x_{1}}(y)\right) \\
& \left.+\left(\frac{\partial F}{\partial x_{1}}(y) e_{1}-e_{1} \frac{\partial F}{\partial x_{1}}(y)\right) e_{1}\right\} d S_{y}
\end{aligned}
$$

(by the hypothesis $\mathbf{D} F-F \mathbf{D}=0$ )

$=0 \quad($ as the integrand is zero $)$. 
The proof of (7.1) with $j \neq k$ is similar (though a little more involved), requiring the use of Gauss's theorem on $(n-2)$-balls.

To prove Theorem 7.1 , let

$$
u_{k}=\int_{\substack{|y|=r \\ y \in \mathbb{R}^{n}}} y_{k}(\phi(y) n(y)-n(y) \phi(y)) d S_{y}-\left(\underline{\phi}(r) e_{k}-e_{k} \underline{\phi}(r)\right)
$$

(which by Lemma 6.2 are independent of $r$ ). By Lemma 7.3 the $u_{k}$ satisfy (7.1). So according to Lemma 7.2, there is a solution $v \in \mathbb{R}_{(n)}$ (or $\mathbb{C}_{(n)}$ ) satisfying $v e_{k}-e_{k} v=u_{k}$ and $|v| \leq C \sum_{k=1}^{n}\left|u_{k}\right| \leq C\|(\phi, \underline{\phi})\|_{K\left(S_{\mu}^{o}\right)}$. It is easy to check that, for this $v$,

$$
\int_{\substack{|y|=r \\ y \in \mathbb{R}^{n}}}\langle y, x\rangle(\phi(y) n(y)-n(y) \phi(y)) d y-((\underline{\phi}(r)+v) x-x(\underline{\phi}(r)+v))=0
$$

which means that $(\phi, \underline{\phi}+v) \in M\left(S_{\mu}^{o}\right)$.

\section{REFERENCES}

1. F. Brackx, R. Delanghe, and F. Sommen, Clifford analysis, Res. Notes in Math., no. 76, Pitman Boston, 1982.

2. A. P. Calderón, Cauchy integrals on Lipschitz curves and related operators, Proc. Nat. Acad. Sci. U.S.A. 74 (1977), 1324-1327.

3. R. R. Coifman, A. McIntosh, and Y. Meyer, L'intégrale de Cauchy définit un opérateur borné sur $L^{2}$ pour les courbes lipschitziennes, Ann. of Math. (2) 116 (1982), 361-387.

4. R. R. Coifman and Y. Meyer, Fourier analysis of multilinear convolutions, Calderón's theorem, and analysis on Lipschitz curves, Lecture Notes in Math., vol. 779, Springer-Verlag, 1980, pp. 104-122.

5. R. R. Coifman, P. Jones, and S. Semmes, Two elementary proofs of the $L^{2}$ boundedness of Cauchy integrals on Lipschitz curves, Amer. Math. Soc. 2 (1989), 553-564.

6. B. E. J. Dahlberg, Poisson semigroups and singular integrals, Proc. Amer. Math. Soc. 97 (1986), 41-48.

7. G. David, J. L. Journé, and S. Semmes, Opérateurs de Calderón-Zygmund, fonctions paraaccrétives et interpolation, Rev. Math. Iberoamer. 1 (1985), 1-57.

8. G. I. Gaudry, R. L. Long, and T. Qian, A martingale proof of $L^{2}$-boundedness of Clifford-valued singular integrals, Ann. Mat. Pura Appl. (to appear).

9. J. E. Gilbert and M. A. M. Murray, $H^{p}$-Theory on Euclidean space and the Dirac operator, Rev. Mat. Iberoamer. 4 (1988), 253-289.

10. V. Iftimie, Fonctions hypercomplexes, Bull. Math. Soc. Sci. Math. R. S. Roumanie (N.S.) 9 (1965), 279-332.

11. C. Kenig, Weighted $H^{p}$ spaces on Lipschitz domains, Amer. J. Math. 102 (1980), 129-163.

12. C. Li, A. McIntosh, and T. Qian, Clifford algebras, Fourier transforms, and singular convolution operators on Lipschitz surfaces, submitted for publication.

13. A. McIntosh, Clifford algebras and the higher dimensional Cauchy integral, Approximation Theory and Function Spaces, Banach Center Publ., no. 22, PWN, Warsaw, 1989, pp. 253-267.

14. A. McIntosh and T. Qian, Fourier theory on Lipschitz curves, Miniconference on Harmonic Analysis, 1987, Proc. Centre Math. Anal. Austral. Nat. Univ., no. 15, Austral. Nat. Univ., Canberra, 1987, pp. 157-166.

15. A. McIntosh and T. Qian, Convolution singular integral operators on Lipschitz curves, Lecture Notes in Math., vol. 1494, Springer-Verlag, 1991, pp. 142-162. 
16. Y. Meyer, Ondelettes et opérateurs. II: Opérateurs de Calderón-Zygmund, Hermann, Paris, 1990.

17. M.A.M. Murray, The Cauchy integral, Calderón commutators and conjugations of singular integrals in $\mathbb{R}^{m}$, Trans. Amer. Math. Soc. 289 (1985), 497-518.

Abstract. We prove the $L_{p}$-boundedness of convolution singular integral operators on a Lipschitz surface

$$
\Sigma=\left\{g(\mathbf{x}) e_{0}+\mathbf{x} \in \mathbb{R}^{n+1}: \mathbf{x} \in \mathbb{R}^{n}\right\}
$$

where $g$ is a Lipschitz function which satisfies $\|\nabla g\|_{\infty} \leq \tan \omega<\infty$. Here we have embedded $\mathbb{R}^{n+1}$ in the Clifford algebra $\mathbb{R}_{(n)}$ with identity $e_{0}$, and are considering convolution with right-monogenic functions $\phi$ which satisfy $|\phi(x)| \leq C|x|^{-n}$ on a sector

$$
S_{\mu}^{o}=\left\{x=x_{0}+\mathbf{x} \in \mathbb{R}^{n+1}:\left|x_{0}\right|<|\mathbf{x}| \tan \mu\right\}
$$

where $\mu>\omega$. Provided there exists an $L_{\infty}$ function $\underline{\phi}$ satisfying

$$
\underline{\phi}(R)-\underline{\phi}(r)=\int_{\substack{r<|x|<R \\ x \in \mathbb{R}^{n}}} \phi(x) d x
$$

then the related convolution singular integral operator

$$
\left(T_{(\phi, \underline{\phi})} u\right)(x)=\lim _{\varepsilon \rightarrow 0+}\left\{\int_{|x-y| \geq \varepsilon}^{y \in \Sigma} \phi(x-y) n(y) u(y) d S_{y}+\underline{\phi}(\varepsilon n(x)) u(x)\right\}
$$

is bounded on $L_{p}(\Sigma)$ for $1<p<\infty$.

(Chun Li and Alan McIntosh) School of Mathematics, Physics, Computing, and Electronics, Macquarie University, NSW 2109, Australia

E-mail address, Chun Li: chun@macadam.mpce.mq.edu.au

E-mail address, Alan McIntosh: alan@macadam.mpce.mq.edu.au

(Stephen Semmes) Department of Mathematics, Rice University, Houston, TeXas 77251

E-mail address: semmes@rice.edu 